\title{
Effectiveness and safety of structured exercise vs. no exercise for asymptomatic aortic aneurysm: systematic review and meta-analysis
}

\author{
Efetividade e segurança de exercícios estruturados versus sem exercício em pacientes \\ assintomáticos com aneurisma de aorta: revisão sistemática e metanálise \\ Ricardo de Ávila Oliveira ${ }^{1,2}$ (D), Eliza Nakajima² (D), Vladimir Tonello de Vasconcelos² (D), Rachel Riera² (D), \\ José Carlos Costa Baptista-Silva²
}

\begin{abstract}
We conducted a systematic review to compare the effectiveness and safety of exercise versus no exercise for patients with asymptomatic aortic aneurysm. We followed the guidelines set out in the Cochrane systematic review handbook. We searched Medline, Embase, CENTRAL, LILACS, PeDRO, CINAHL, clinicaltrials.gov, ICTRP, and OpenGrey using the MeSH terms "aortic aneurysm" and "exercise". 1189 references were identified. Five clinical trials were included. No exercise-related deaths or aortic ruptures occurred in these trials. Exercise did not reduce the aneurysm expansion rate at 12 weeks to 12 months (mean difference [MD], -0.05 ; 95\% confidence interval [CI], -0.13 to 0.03 ). Six weeks of preoperative exercise reduced severe renal and cardiac complications (risk ratio, $0.54 ; 95 \% \mathrm{Cl}, 0.31-0.93$ ) and the length of intensive care unit stay $(\mathrm{MD},-1.00 ; 95 \% \mathrm{Cl},-1.26$ to -0.74$)$. Preoperative and postoperative forward walking reduced the length of hospital stay $(\mathrm{MD},-0.69 ; 95 \% \mathrm{Cl},-1.24$ to -0.14$)$. The evidence was graded as 'very low' level.
\end{abstract}

Keywords: aortic aneurysm; abdominal aortic aneurysm; exercise; postoperative complications.

\begin{abstract}
Resumo
Foi realizada revisão sistemática para comparar a efetividade e a segurança de exercícios versus não exercícios em pacientes assintomáticos com aneurisma de aorta. Usamos os termos MeSH aortic aneurysm e exercise para as bases MEDLINE, Embase, CENTRAL, LILACS, PeDRO, CINAHL, clinicaltrials.gov, International Clinical Trials Registry Platform (ICTRP) e OpenGrey. Foram obtidas 1.189 referências. Cinco ensaios clínicos foram incluídos. Não houve morte ou rotura associada ao exercício. Além disso, este não reduziu a velocidade de crescimento do aneurisma em 12 semanas a 12 meses [diferença de médias (DM) -0,05; intervalo de confiança de 95\% (IC95\%) -0,13 a 0,03]. Seis semanas de exercícios pré-operatórios reduziram complicações clínicas renais e cardíacas (razão de risco 0,54; IC95\% 0,31-0,93) e a permanência em unidade de terapia intensiva ( $D M-1,00$; IC95\% -1,26 a -0,74). Caminhadas nos períodos pré e pós-operatório reduziram a permanência hospitalar. A evidência foi classificada como de muito baixa qualidade.
\end{abstract}

Palavras-chave: aneurismas de aorta; aneurismas de aorta abdominal; exercícios; complicações pós-operatórias.

How to cite: Oliveira RÁ, Nakajima E, Vasconcelos VT, Riera R, Baptista-Silva JCC. Effectiveness and safety of structured exercise vs. no exercise for asymptomatic aortic aneurysm: systematic review and meta-analysis. I Vasc Bras. 2020;19:e20190086. https://doi.org/10.1590/1677-5449.190086

\footnotetext{
${ }^{1}$ Universidade Federal de Uberlândia - UFU, Departamento de Cirurgia, Uberlândia, MG, Brasil. ${ }^{2}$ Universidade Federal de São Paulo - UNIFESP, Departamento de Medicina, São Paulo, SP, Brasil. Financial support: None.

Conflicts of interest: No conflicts of interest declared concerning the publication of this article. Submitted: August 15, 2019. Accepted: October 01, 2019.
} 


\section{INTRODUCTION}

An aortic aneurysm is a permanent localized aortic dilatation that is at least $50 \%$ larger than the normal diameter. ${ }^{1,2}$ The estimated prevalence is $4.0 \%$ to $8.9 \%$ in men and $1.3 \%$ to $2.2 \%$ in women aged $\geq 55$ years. ${ }^{3,4}$ Approximately $80 \%$ of aortic aneurysms are located in the abdominal aorta. ${ }^{5}$ They usually have an asymptomatic natural history and so diagnosis is made after thorough investigation. ${ }^{6}$ The most feared complication of aortic aneurysms is rupture, which leads to death in up to $90 \%$ of patients. ${ }^{7}$ The risk of rupture increases as the aneurysm diameter increases. ${ }^{2,8,9}$ Surgery is recommended when an aneurysm reaches $50 \mathrm{~mm}$ in women or $55 \mathrm{~mm}$ in men, ${ }^{10}$ because at this point the risks of surveillance outweigh the risks of surgery. No clinical interventions have been found to be effective for reducing the growth rate or risk of rupture before an aneurysm reaches these diameters. ${ }^{10,11}$

In one study, $87.7 \%$ of the patients diagnosed had aneurysms with diameters of $<3.5 \mathrm{~cm}$. ${ }^{12}$ Therefore, despite the tremendous effort that has been expended on surgical research, there is not enough information to recommend non-pharmacologic clinical treatment for most patients, other than smoking cessation and controlling blood pressure. Even among patients with small aneurysms, the most frequent cause of death is myocardial infarction and stroke, not aneurysm rupture. ${ }^{10,12}$ Moreover, when patients do undergo surgery, $41 \%$ of deaths are also related to cardiovascular events. ${ }^{13}$ Therefore, an aortic aneurysm is a risk factor for death and has a risk of mortality $50 \%$ higher than that in persons with no aortic pathology. ${ }^{12}$

Exercise and smoking cessation help to reduce mortality and improve quality of life. ${ }^{14,15}$ Exercise is a subgroup of physical activity defined as planned, structured, and repetitive activities performed with the objective of improving or maintaining physical fitness. ${ }^{16}$ Despite the importance of exercise, there is no consensus regarding exercise recommendations for patients with aortic aneurysms, ${ }^{17,18}$ because of the fear of aneurysm rupture and doubts about the effectiveness of exercise. Hence, a systematic review of the literature is crucial to describe the risks and benefits of exercise for patients with aortic aneurysms.

The study was performed to assess the effectiveness and safety of exercise for asymptomatic patients with an aortic aneurysm.

\section{MATERIALS AND METHODS}

This review was conducted in the Post-graduate Program in Evidence-based Healthcare at the Universidade Federal de São Paulo (UNIFESP), São Paulo, SP, Brazil. It followed the recommendations contained in the Cochrane Handbook for Systematic Reviews of Interventions, ${ }^{19}$ and reporting of the results complies with the PRISMA Statement for quality in publication. ${ }^{20}$ The review protocol was registered on the PROSPERO database. ${ }^{21}$ The review was also approved by the institutional ethics committee (CAAE number: 57716016.0.0000.5505).

Randomized and quasi-randomized clinical trials were considered for inclusion. Due to the nature of the intervention, crossover studies were not considered for this review.

The inclusion criteria were sedentary patients (those performing only daily activities during the last year), adults ( $\geq 18$ years of age), and the presence of an aortic aneurysm confirmed by a diagnostic imaging examination.

The exclusion criteria were rapid growth of aneurysms $(0.5 \mathrm{~cm}$ within 6 months or $1.0 \mathrm{~cm}$ within 1 year), saccular aneurysms, complicated aneurysms (such as symptomatic, completely thrombosed, or ruptured aneurysms), and inflammatory and infectious aneurysms. High intensity interval training exercises were excluded.

Any exercise was considered (individual or in groups, assisted or self-managed, aerobic, stretching or strengthening; any intensity, frequency, and duration; and alone or combined with any other intervention), as long as the same co-intervention was also performed in the comparison group. This group of participants was designated the exercise group. For the purposes of the present study, exercise was defined as a subgroup of physical activity that is planned, structured, and repetitive and aims to improve or maintain one or more components of physical fitness. ${ }^{16}$ The comparators considered in this review were patients receiving no intervention and patients on a waiting list. If a study compared different types of exercises (e.g., strength exercises versus resistance exercises), we considered performing a comparison of exercises versus advice for exercising or a different type of exercise used. These patients were designated the no exercise group.

\section{Primary outcomes}

- All-cause mortality in the short-term (up to 30 days after beginning exercise) and long-term (from 30 days to $\geq 1$ year after starting exercise);

- Number of participants presenting with aneurysmal rupture;

- Aneurysm growth rate (change, in millimeters $(\mathrm{mm})$, in the aneurysm diameter from baseline to the end of the study). 


\section{Secondary outcomes}

- Quality of life, measured by any validated tool;

- Number of participants referred for aneurysm surgery;

- Number of participants presenting with at least one severe short-term (up to 24 hours after surgery), intermediate-term (from 24 hours to 30 days after surgery), or long-term ( $>30$ days after surgery) complication. A severe complication was defined as myocardial infarction, prolonged inotropic support, new-onset arrhythmia, unstable angina, postoperative pneumonia, unexplained re-intubation, or renal insufficiency (requirement for dialysis or a $>20 \%$ reduction in creatinine clearance);

- Hospital stay related to aneurysm surgery (in days);

- Intensive care unit stay after aneurysm surgery (in days);

- Forced expiratory volume in 1 second as measured with a spirometer.

Any outcome not mentioned in the protocol was described as a non-proposed outcome in the results.

The following electronic databases were searched and updated: Literatura Latino Americana em Ciências da Saúde e do Caribe (LILACS) (via the Biblioteca Virtual em Saúde [BVS], from 1966 to 13 December 2018), Medline (via PubMed, from inception to 13 December 2018), Cochrane Central Register of Controlled Trials (CENTRAL) (via Wiley Cochrane Library, December 2018 Edition), Embase (via Elsevier, from 1974 to 13 December 2018), PEDro (via BVS, from inception to November 2018), and Cumulative Index to Nursing and Allied Health Literature (CINAHL) (EBSCO, from inception to 7 November 2018). Additional searches were conducted on the trial registry databases ClinicalTrials.gov, the World Health Organization International Clinical Trials Registry Platform (ICTRP) search portal, and the gray literature (http://www.opengrey.eu/) (from inception to 13 December 2018). A manual search was also performed of the reference lists of all studies included and relevant systematic reviews.

There were no search limits for data, status, or language of publication. The search strategy for Medline is shown in Table 1.

Two reviewers independently screened the titles and abstracts for selection and inclusion using Rayyan software. ${ }^{22}$ They also extracted data and assessed the methodological quality of the studies included as described in the PROSPERO registry database..$^{21} \mathrm{~A}$ third reviewer resolved any disagreements at each stage.

The strategies for data synthesis, meta-analysis, effect size, subgroup, and sensitivity analysis are also described in the PROSPERO database. ${ }^{21}$ RevMan 5.3 software ${ }^{23}$ was used to measure the effect size and perform a meta-analysis when possible. A funnel plot was also planned as part of the protocol.

The GRADE approach was used to evaluate the quality of the body of evidence. ${ }^{24}$ Each decision to downgrade the quality of studies was justified (Tables 2, 3, 4, 5, and 6). A summary-of-findings table was created using GRADEpro GDT considering the primary outcomes and the main comparisons (exercise vs. no exercise at 7- to 12-week surveillance and at 3 years; exercise vs. no exercise before surgery; exercise vs. no exercise after surgery; and exercise vs. no exercise before and after surgery). ${ }^{24}$ The outcomes were death, aortic rupture, aneurysm growth rate, number of patients with at least one cardiovascular complication, and number of patients referred for surgery.

\section{RESULTS}

The search strategy returned 1189 references (Figure 1). From these, 8 references from 5 clinical trials involving a total of 387 participants were included

Table 1. MEDLINE search strategy.

("Aortic Aneurysm"[Mesh] OR (Aortic Aneurysm) OR (Aneurysms, Aortic) OR (Aortic Aneurysms) OR (Aneurysm, Aortic)) AND ((“Exercise”[Mesh]) OR (Exercise) OR (Exercises) OR (Exercise, Physical) OR (Exercises, Physical) OR (Physical Exercise) OR (Physical Exercises) OR (Exercise, Isometric) OR (Exercises, Isometric) OR (Isometric Exercises) OR (Isometric Exercise) OR (Exercise, Aerobic) OR (Aerobic Exercises) OR (Exercises, Aerobic) OR (Aerobic Exercise) OR "Physical Fitness"[Mesh] OR (Fitness, Physical) OR (Physical

MEDLINE via PubMed Fitness) OR "Exercise Therapy"[Mesh] OR (Therapy, Exercise) OR (Exercise Therapies) OR (Therapies, Exercise) search strategy OR "Physical Exertion" [Mesh] OR (Exertion, Physical) OR (Exertions, Physical) OR (Physical Exertions) OR (Physical Effort) OR (Effort, Physical) OR (Efforts, Physical) OR (Physical Efforts) OR "Sports"[Mesh] OR (Sport) OR (Athletics) OR (Athletic) OR "Exercise Movement Techniques"[Mesh] OR (Movement Techniques, Exercise) OR (Exercise Movement Technics) OR (Pilates-Based Exercises) OR (Exercises, Pilates-Based) OR (Pilates Based Exercises) OR (Pilates Training) OR (Training, Pilates) OR “Physical Endurance"[Mesh] OR (Endurance, Physical) OR (Endurances, Physical) OR (Physical Endurances)) 
Table 2. GRADEpro-GDT judgment of the quality of evidence: GRADE question: Should exercise be indicated for patients with aortic aneurysms at surveillance?

Summary of findings:

Exercise compared to no exercise for aortic aneurysm patients at surveillance in intermediate term (7-12 w)

Patient or population: aortic aneurysm patients at surveillance in intermediate term (7-12 $\mathrm{w})$

Setting:

Intervention: exercise

Comparison: no exercise

\begin{tabular}{|c|c|c|c|c|c|}
\hline \multirow[b]{2}{*}{ Outcomes } & \multicolumn{2}{|c|}{ Anticipated absolute effects* $(95 \% \mathrm{CI})$} & \multirow{2}{*}{$\begin{array}{l}\text { Relative effect } \\
\qquad(95 \% \mathrm{Cl})\end{array}$} & \multirow{2}{*}{$\begin{array}{l}\mathrm{N}^{\circ} \text { of } \\
\text { participants } \\
\text { (studies) }\end{array}$} & \multirow{2}{*}{$\begin{array}{l}\text { Certainty of the } \\
\text { evidence } \\
\text { (GRADE) }\end{array}$} \\
\hline & $\begin{array}{l}\text { Risk with no } \\
\text { exercise }\end{array}$ & Risk with exercise & & & \\
\hline $\begin{array}{l}\text { Mortality } \\
\text { follow up: range } 7 \text { weeks to } 12 \text { weeks }\end{array}$ & 0 per 100 & $\begin{array}{l}0 \text { per } 100 \\
(0 \text { to } 0)\end{array}$ & not estimable & $\begin{array}{c}263 \\
(4 \mathrm{RCTs})\end{array}$ & $\begin{array}{c}\oplus \bigcirc \bigcirc \bigcirc \\
\text { VERY LOW }\end{array}$ \\
\hline $\begin{array}{l}\text { Aortic rupture } \\
\text { follow up: range } 7 \text { weeks to } 12 \text { weeks }\end{array}$ & 0 per 100 & $\begin{array}{l}0 \text { per } 100 \\
(0 \text { to } 0)\end{array}$ & not estimable & $\begin{array}{c}263 \\
(4 \mathrm{RCTs})\end{array}$ & $\begin{array}{l}\oplus \bigcirc \bigcirc \bigcirc \\
\text { VERY LOW }\end{array}$ \\
\hline $\begin{array}{l}\text { Aneurysm growth rate } \\
\text { follow up: range } 7 \text { weeks to } 12 \text { weeks }\end{array}$ & $\begin{array}{l}\text { The mean } \\
\text { aneurysm } \\
\text { growth rate } \\
\text { was } 0\end{array}$ & $\begin{array}{l}\text { The mean aneurysm growth } \\
\text { rate in the exercise group } \\
\text { was } 0.06 \text { lower ( } 0.23 \text { lower } \\
\text { to } 0.11 \text { higher) }\end{array}$ & - & (2 RCTs) & $\overbrace{\text { VERY LOW }}^{\oplus, c, d, e}$ \\
\hline $\begin{array}{l}\text { Number of patients with at least one } \\
\text { cardiovascular complication } \\
\text { follow up: range } 7 \text { weeks to } 12 \text { weeks }\end{array}$ & 0 per 100 & $\begin{array}{l}0 \text { per } 100 \\
(0 \text { to } 0)\end{array}$ & $\begin{array}{c}\text { RR } 100.00 \\
(0.07 \text { to } 35.46)\end{array}$ & $\begin{array}{c}263 \\
(4 \mathrm{RCTs})\end{array}$ & $\begin{array}{l}\oplus \bigcirc \bigcirc \bigcirc \\
\text { VERY LOW }\end{array}$ \\
\hline $\begin{array}{l}\text { Number of patients who reached } \\
\text { threshold for surgery } \\
\text { follow up: range } 7 \text { weeks to } 12 \text { weeks }\end{array}$ & 0 per 100 & $\begin{array}{l}0 \text { per } 100 \\
(0 \text { to } 0)\end{array}$ & not estimable & $\begin{array}{c}263 \\
(4 \mathrm{RCTs})\end{array}$ & $\begin{array}{l}\oplus \bigcirc \bigcirc \bigcirc \\
\text { VERY LOW }\end{array}$ \\
\hline
\end{tabular}

*The risk in the exercise group (and its $95 \%$ confidence interval) is based on the assumed risk in the comparison group and the relative effect of the intervention (and its 95\% Cl); Cl: Confidence interval; MD: Mean difference; RR: Risk ratio; GRADE Working Group grades of evidence. High certainty: We are very confident that the true effect lies close to that of the estimate of the effect. Moderate certainty: We are moderately confident in the effect estimate: The true effect is likely to be close to the estimate of the effect, but there is a possibility that it is substantially different. Low certainty: Our confidence in the effect estimate is limited: The true effect may be substantially different from the estimate of the effect. Very low certainty: We have very little confidence in the effect estimate: The true effect is likely to be substantially different from the estimate of effect. Explanations: a. Half of the studies did not have blinded outcome assessment or did not have allocation concealment or the randomization method was unclear; b. The time point of measurement was not long enough to support any conclusions; c. Low number of events; $d$. Small sample size; e. The study had unblinded outcome assessment and did not have allocation concealment. The randomization method was unclear and there were incomplete outcome data.

Table 3. GRADEpro-GDT judgment of the quality of the evidence: GRADE question: Should exercise be indicated for patients with aortic aneurysms at surveillance?

Summary of findings:

Exercises compared to no exercise for aortic aneurysm patients at surveillance in long term (at 3 y)

Patient or population: aortic aneurysm patients at surveillance in long term (at $3 \mathrm{y}$ )

Setting:

Intervention: exercise

Comparison: no exercise

\begin{tabular}{|c|c|c|c|c|c|}
\hline \multirow[b]{2}{*}{ Outcomes } & \multicolumn{2}{|c|}{ Anticipated absolute effects* $(95 \% \mathrm{Cl})$} & \multirow{2}{*}{$\begin{array}{l}\text { Relative effect } \\
\qquad(95 \% \mathrm{Cl})\end{array}$} & \multirow{2}{*}{$\begin{array}{l}\text { No of participants } \\
\text { (studies) }\end{array}$} & \multirow{2}{*}{$\begin{array}{l}\text { Certainty of the } \\
\text { evidence (GRADE) }\end{array}$} \\
\hline & $\begin{array}{l}\text { Risk with no } \\
\text { exercise }\end{array}$ & Risk with exercise & & & \\
\hline $\begin{array}{c}\text { Mortality } \\
\text { follow up: mean } 3 \text { years }\end{array}$ & 0 per 100 & $\begin{array}{l}0 \text { per } 100 \\
(0 \text { to } 0)\end{array}$ & not estimable & $\begin{array}{c}140 \\
(1 \mathrm{RCT})\end{array}$ & $\begin{array}{l}\oplus \bigcirc \bigcirc \bigcirc \\
\text { VERY LOW }\end{array}$ \\
\hline $\begin{array}{c}\text { Aortic rupture follow up: } \\
\text { mean } 3 \text { years }\end{array}$ & 0 per 100 & $\begin{array}{c}0 \text { per } 100 \\
(0 \text { to } 0)\end{array}$ & not estimable & $\begin{array}{c}45 \\
(1 \mathrm{RCT})\end{array}$ & $\begin{array}{l}\oplus \bigcirc \bigcirc \bigcirc \\
\text { VERY LOW }\end{array}$ \\
\hline $\begin{array}{l}\text { Aneurysm growth rate } \\
\text { follow up: mean } 3 \text { years }\end{array}$ & $\begin{array}{l}\text { The mean } \\
\text { aneurysm growth } \\
\text { rate was } 0.54\end{array}$ & $\begin{array}{l}\text { The mean aneurysm } \\
\text { growth rate in the exercise } \\
\text { group was } 0.06 \text { lower } \\
\text { (0.23 lower to } 0.11 \text { higher) }\end{array}$ & - & $\begin{array}{c}45 \\
(1 \mathrm{RCT})\end{array}$ & $\overbrace{\text { VERY LOW }}^{\oplus \bigcirc, b, c, d, e}$ \\
\hline $\begin{array}{l}\text { Number of patients with at least } \\
\text { one cardiovascular complication } \\
\text { follow up: mean } 3 \text { years }\end{array}$ & 0 per 100 & $\begin{array}{l}0 \text { per } 100 \\
(0 \text { to } 0)\end{array}$ & not estimable & $\begin{array}{c}45 \\
(1 \mathrm{RCT})\end{array}$ & $\underbrace{\oplus, b, c, d, e}_{\text {VERY LOW }}$ \\
\hline $\begin{array}{c}\text { Number of patients who reached } \\
\text { threshold for surgery follow up: } \\
\text { mean } 3 \text { years }\end{array}$ & 13 per 100 & $\begin{array}{l}4 \text { per } 100 \\
(1 \text { to } 15)\end{array}$ & $\begin{array}{c}\text { RR } 0.31 \\
(0.09 \text { to } 1.11)\end{array}$ & $\begin{array}{c}140 \\
(1 \mathrm{RCT})\end{array}$ & $\overbrace{\text { VERY LOW }}^{\oplus \bigcirc, \mathrm{b}, \mathrm{c}, \mathrm{d}, \mathrm{e}}$ \\
\hline
\end{tabular}

*The risk in the exercise group (and its $95 \%$ confidence interval) is based on the assumed risk in the comparison group and the relative effect of the intervention (and its 95\% Cl). Cl: Confidence interval; MD: Mean difference; RR: Risk ratio. GRADE Working Group grades of evidence. High certainty: We are very confident that the true effect lies close to that of the estimate of the effect. Moderate certainty: We are moderately confident in the effect estimate: The true effect is likely to be close to the estimate of the effect, but there is a possibility that it is substantially different. Low certainty: Our confidence in the effect estimate is limited: The true effect may be substantially different from the estimate of the effect. Very low certainty: We have very little confidence in the effect estimate: The true effect is likely to be substantially different from the estimate of effect. Explanations a. The randomization method, allocation concealment, blinding of outcome assessment, and other sources of bias are unclear. There is a high risk of incomplete outcome data; $b$. The intervention was performed in a very controlled setting, not applied to the usual patient; c. There was only one study; $\mathrm{d}$. The sample size is too small to make a judgment; e. The number of events were small; f. Single study. Large number of patients lost to follow-up. 
Table 4. GRADEpro-GDT judgment of the quality of the evidence: GRADE question: Should exercise be indicated for patients with aortic aneurysms before surgery?

\begin{tabular}{|c|c|c|c|c|c|c|}
\hline \multicolumn{7}{|c|}{ Exercises compared to no exercise for aortic aneurysm patients before surgery } \\
\hline \multicolumn{7}{|c|}{$\begin{array}{l}\text { Patient or population: aortic aneurysm patients before surgery } \\
\text { Setting: } \\
\text { Intervention: exercise } \\
\text { Comparator: no exercise }\end{array}$} \\
\hline \multirow{2}{*}{ Outcomes } & \multicolumn{2}{|c|}{$\begin{array}{l}\text { Anticipated absolute effects* } \\
(95 \% \mathrm{Cl})\end{array}$} & \multirow{2}{*}{$\begin{array}{l}\text { Relative effect } \\
\qquad(95 \% \mathrm{Cl})\end{array}$} & \multirow{2}{*}{$\begin{array}{l}\mathrm{N}^{\circ} \text { of } \\
\text { participants } \\
\text { (studies) }\end{array}$} & \multirow{2}{*}{$\begin{array}{l}\text { Certainty of the } \\
\text { evidence } \\
\text { (GRADE) }\end{array}$} & \multirow{2}{*}{ Comment } \\
\hline & $\begin{array}{l}\text { Risk with no } \\
\text { exercise }\end{array}$ & $\begin{array}{l}\text { Risk with } \\
\text { exercise }\end{array}$ & & & & \\
\hline $\begin{array}{l}\text { Mortality } \\
\text { follow up: mean } \\
30 \text { days }\end{array}$ & 3 per 100 & $\begin{array}{l}3 \text { per } 100 \\
\text { ( } 3 \text { to } 3 \text { ) }\end{array}$ & $\begin{array}{c}\text { RR } 1.00 \\
(0.93 \text { to } 1.07)\end{array}$ & $\begin{array}{c}124 \\
(1 \mathrm{RCT})\end{array}$ & $\begin{array}{l}\bigoplus \bigcirc \bigcirc \bigcirc \\
\text { VERY LOW }\end{array}$ & $\begin{array}{l}\text { Mortality was related } \\
\text { to surgery. There was } \\
\text { no mortality related } \\
\text { to exercise. }\end{array}$ \\
\hline $\begin{array}{l}\text { Aortic rupture } \\
\text { follow up: mean } \\
30 \text { days }\end{array}$ & 0 per 100 & $\begin{array}{l}0 \text { per } 100 \\
(0 \text { to } 0)\end{array}$ & not estimable & $\begin{array}{c}124 \\
(1 \mathrm{RCT})\end{array}$ & $\begin{array}{l}\bigoplus \bigcirc \bigcirc \bigcirc \\
\text { VERY LOW }\end{array}$ & \\
\hline $\begin{array}{l}\text { Aneurysm growth } \\
\text { rate - not measured }\end{array}$ & - & - & - & - & - & \\
\hline $\begin{array}{l}\text { Number of patients } \\
\text { with at least one } \\
\text { cardiovascular } \\
\text { complication } \\
\text { follow up: mean } \\
30 \text { days }\end{array}$ & 23 per 100 & $\begin{array}{l}8 \text { per } 100 \\
(3 \text { to } 21)\end{array}$ & $\begin{array}{c}\text { RR } 0.36 \\
(0.14 \text { to } 0.93)\end{array}$ & $\begin{array}{c}124 \\
(1 \mathrm{RCT})\end{array}$ & $\begin{array}{l}\bigoplus \bigcirc \bigcirc \bigcirc \\
\text { VERY LOW }\end{array}$ & \\
\hline $\begin{array}{l}\text { Number of patients } \\
\text { who reached } \\
\text { threshold for surgery } \\
\text { - not measured }\end{array}$ & - & - & - & - & - & \\
\hline \multicolumn{7}{|c|}{$\begin{array}{l}\text { *The risk in the exercise group (and its 95\% confidence interval) is based on the assumed risk in the comparison group and the relative effect of the intervention (and } \\
\text { its 95\% CI). Cl: Confidence interval; RR: Risk ratio; GRADE Working Group grades of evidence. High certainty: We are very confident that the true effect lies close to } \\
\text { that of the estimate of the effect. Moderate certainty: We are moderately confident in the effect estimate: The true effect is likely to be close to the estimate of the } \\
\text { effect, but there is a possibility that it is substantially different. Low certainty: Our confidence in the effect estimate is limited: The true effect may be substantially } \\
\text { different from the estimate of the effect. Very low certainty: We have very little confidence in the effect estimate: The true effect is likely to be substantially different } \\
\text { from the estimate of effect. Explanations: a. Due to nature of intervention, it was impossible to blind participants and personnel; b. Single study, low number of events }\end{array}$} \\
\hline
\end{tabular}

Table 5. GRADEpro-GDT judgment of the quality of the evidence: GRADE question: Should exercise be indicated for patients with aortic aneurysms after surgery?

Summary of findings:

Exercise compared to no exercise for aortic aneurysm patients after surgery

Patient or population: aortic aneurysm patients after surgery

Setting:

Intervention: exercise

Comparison: no exercise

Outcomes
Mortality - not measured
Aortic rupture - not measured
Aneurysm growth rate - not measured
Number of patients with at least one
cardiovascular complication - not measured
Number of patients who reached threshold
for surgery - not measured
*The risk in the exercise group (and its 95\% confidence interval) is based on the assumed risk in the comparison group and the relative effect of the intervention
(and its $95 \%$ Cl); Cl: Confidence interval. GRADE Working Group grades of evidence. High certainty: We are very confident that the true effect lies close to that of
the estimate of the effect. Moderate certainty: We are moderately confident in the effect estimate: The true effect is likely to be close to the estimate of the effect,
but there is a possibility that it is substantially different. Low certainty: Our confidence in the effect estimate is limited: The true effect may be substantially different
from the estimate of the effect. Very low certainty: We have very little confidence in the effect estimate: The true effect is likely to be substantially different from
(GRADE)
the estimate of effect.


Table 6. GRADEpro-GDT judgment of the quality of the evidence: GRADE question: Should exercise be indicated for patients with aortic aneurysms before and after surgery?

Summary of findings:

Exercises compared to no exercise for aortic aneurysm patients before and after surgery

Patient or population: aortic aneurysm patients before and after surgery

Setting:

Intervention: exercise

Comparison: no exercise

\begin{tabular}{|c|c|c|c|c|c|c|}
\hline \multirow{2}{*}{ Outcomes } & \multicolumn{2}{|c|}{$\begin{array}{l}\text { Anticipated absolute effects* } \\
(95 \% \mathrm{Cl})\end{array}$} & \multirow{2}{*}{$\begin{array}{l}\text { Relative effect } \\
\qquad(95 \% \mathrm{Cl})\end{array}$} & \multirow{2}{*}{$\begin{array}{l}\mathrm{N}^{\circ} \text { of } \\
\text { participants } \\
\text { (studies) }\end{array}$} & \multirow{2}{*}{$\begin{array}{c}\text { Certainty of the } \\
\text { evidence } \\
\text { (GRADE) }\end{array}$} & \multirow{2}{*}{ Comments } \\
\hline & $\begin{array}{l}\text { Risk with usual } \\
\text { care }\end{array}$ & $\begin{array}{l}\text { Risk with physical } \\
\text { exercises }\end{array}$ & & & & \\
\hline
\end{tabular}

Mortality - not measured

Aortic rupture - not measured

Aneurysm growth rate - not measured

Number of patients with at least one

cardiovascular complication - not

measured

Number of patients who reached

threshold for surgery - not measured

*The risk in the exercise group (and its 95\% confidence interval) is based on the assumed risk in the comparison group and the relative effect of the intervention (and its 95\% Cl). Cl: Confidence interval. GRADE Working Group grades of evidence. High certainty: We are very confident that the true effect lies close to that of the estimate of the effect. Moderate certainty: We are moderately confident in the effect estimate: The true effect is likely to be close to the estimate of the effect, but there is a possibility that it is substantially different. Low certainty: Our confidence in the effect estimate is limited: The true effect may be substantially different from the estimate of the effect. Very low certainty: We have very little confidence in the effect estimate: The true effect is likely to be substantially different from the estimate of effect.

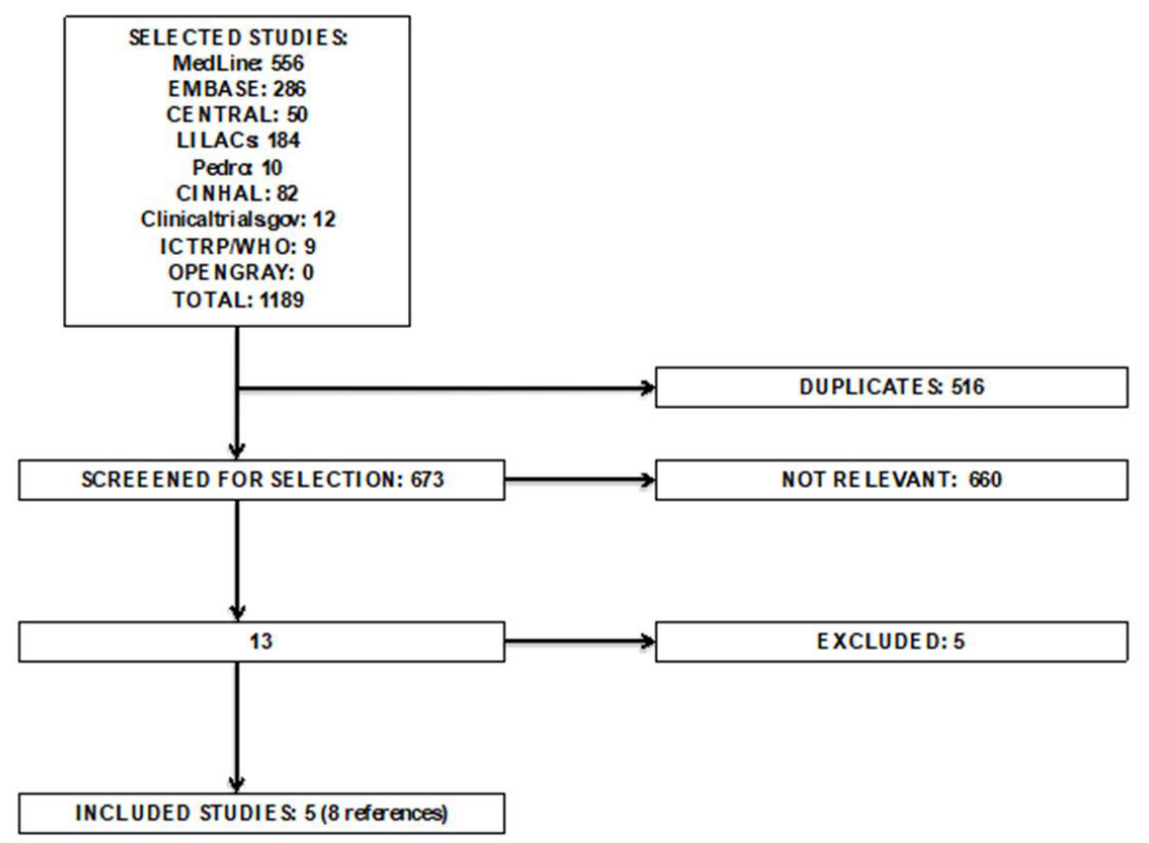

Figure 1. PRISMA flow chart for the review. This figure shows the PRISMA flow chart illustrating the processing of searching for, selecting, excluding, and including studies. There were three references from the same studies: two ${ }^{25,26}$ from Myers et al. ${ }^{11}$ and one ${ }^{27}$ from Barakat et al., ${ }^{28}$ resulting in eight references from five original studies.

(212 participants in exercise groups and 175 in no exercise groups). $)^{11,18,28-30}$ Three studies were conducted during the surveillance period. ${ }^{11,18,29}$ One study was conducted in the preoperative period (preoperative study) ${ }^{28}$ Finally, one study was performed in both the preoperative and postoperative periods (preoperative and postoperative study). ${ }^{30}$ The clinical trial authors also published three other articles based on the same studies: two ${ }^{25,26}$ from Myers et al. ${ }^{11}$ and one ${ }^{27}$ from Barakat et al. ${ }^{28}$

Table 7 describes the studies and their characteristics, including the reasons for inclusion or exclusion. 
Table 7. Studies and characteristics.

\begin{tabular}{|c|c|}
\hline Included studies: & Characteristics: \\
\hline $\begin{array}{c}\text { Kothmann et al. } \\
(2009)^{29}\end{array}$ & $\begin{array}{l}\text { Number of patients in exercise group: } 20 \\
\text { Number of patients in control group: } 10 \\
\text { Age (mean): exercise group: } 69.5 \text { years, control group: } 69.4 \text { years } \\
\text { Time of intervention: At surveillance } \\
\text { Sex: } 20 \text { men and } 5 \text { women } \\
\text { Interventions: \#"Exercise sessions of } 30 \text { min exercise on static Life Fitness bicycle with a } 5 \text { min warm-up and cooling down period. } \\
\text { Participants attended twice weekly in groups of } 3 \text { to } 4 \text { patients. } \\
\text { Participants were required to exercise in zones } 12 \text { to } 14 \text { on the Borg scale."29 } \\
\text { Outcomes: change in anaerobic threshold } \\
\text { Follow-up: } 7 \text { weeks }\end{array}$ \\
\hline
\end{tabular}

Tew et al. (2012) ${ }^{18} \quad$ Number of patients in exercise group: 14

Number of patients in control group: 14

Age: exercise group: $71 \pm 8$ years, control group: $74 \pm 6$ years.

Time of intervention: at surveillance

Sex: male/female: Exercise group: 10/1. Control group: 11/3

Interventions: **"monitored physical exercises three times a week for 35 to 45 minutes at clinical unit; static Life Fitness bicycle in groups of 3 to 4 patients; treadmill walking; to expend up to 2000 Kcal.wk ${ }^{-1 .}$

Borg perceived exhaustion scale at zones of 12 to $14^{18}$.

Outcomes: **"anaerobic threshold; quality of life; safety; and blood markers (including C reactive protein, matrix metalloproteinase-9, and glycemia)"

Follow-up: 12 weeks

Myers et al. Number of patients in exercise group: 72

$(2014)^{11} \quad$ Number of patients in control group: 68

Age: exercise group: $71.8 \pm 7$ years, control group: $71.3 \pm 8$ years.

Time of intervention: at surveillance

Sex: exercise group: $92 \%$ men, control group: $93 \%$ men.

Interventions: $\dagger \dagger$ "monitored physical exercises three times a week workout for 45 minutes at clinical unit or home or both locations; including static Life Fitness bicycle in groups of 3 to 4 patients; treadmill walking; stair climbing; elliptical training; rowing; to expend up to 2000 Kcal.wk'."

Borg perceived exhaustion scale at zones of 12 to $14^{11}$.

Outcomes: safety; aneurysm growth rates.

Follow-up: up to 36 months

Barakat et al. Number of patients in exercise group: 62

$(2016)^{28} \quad$ Number of patients in control group: 62

Age: exercise group: 73.8 years, control group: 72.9 years.

Time of intervention: during preoperative period.

Sex: exercise group: 6 women; control group: 7 women.

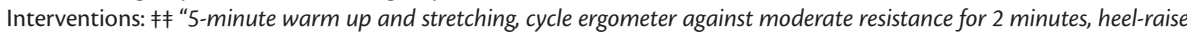
repetitions for 2 minutes, knee extensions against resistance repetitions for 2 minutes, dumbbells' biceps/arm curls repetitions for 2 minutes, step-up lunges repetitions for 2 minutes, knee bends (bodyweight) repetitions for 2 minutes, and 5 minutes for cool down and stretching. Between each of the exercise stations, patients either walked around the gym or on a treadmill or rested for 2 minutes before moving on to the next exercise."

Outcomes: composite postoperative cardiac, renal and postoperative respiratory complications; length of hospital stay, and ITU stay, "APACHE II scores recorded at HDU/ITU admission, the occurrence of systemic inflammatory response syndrome (SIRS), 30-day mortality, postoperative bleeding requiring reoperation or transfusion of more than 4 units of blood products within 72 hours, and the need for reoperation." Follow-up: 12 weeks

Wnuk et al. Number of patients in exercise group: 44 (22 backward walking; 22 forward walking)

$(2016)^{30} \quad$ Number of patients in control group: 21

Age: exercise group: $71 \pm 8$ years, control group: $74 \pm 6$ years.

Time of intervention: during postoperative period.

Sex ratio: male/female: Exercise group: 10/1. Control group: 11/3.

Interventions: postoperative backward walking training, and postoperative forward walking training.

Outcomes: six minutes walking test; heart rate training; standard metabolic equivalent; FVC, FEV1, FEV1/FVC, PEF, hospital-stay. Follow-up: 7 days

\begin{tabular}{|c|c|}
\hline $\begin{array}{l}\text { Excluded } \\
\text { studies: }\end{array}$ & Reasons for exclusion: \\
\hline $\begin{array}{l}\text { Dronkers et al. } \\
(2008)^{31}\end{array}$ & \\
\hline \multicolumn{2}{|c|}{ 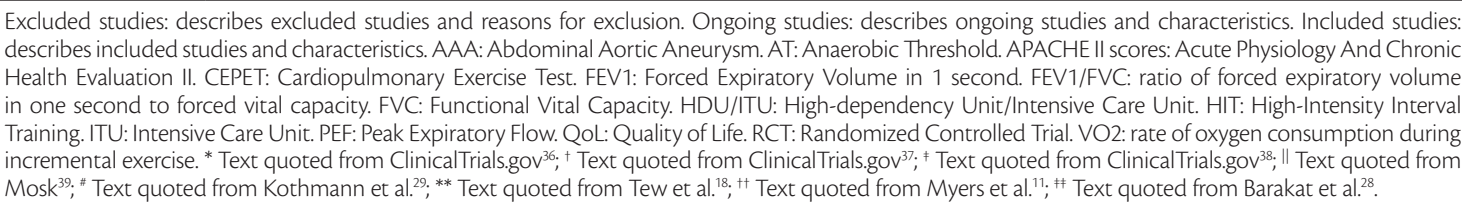 } \\
\hline
\end{tabular}


Table 7. Continued...

\begin{tabular}{|c|c|}
\hline $\begin{array}{l}\text { Excluded } \\
\text { studies: }\end{array}$ & Reasons for exclusion: \\
\hline $\begin{array}{l}\text { Nakayama et al. } \\
\qquad(2018)^{32}\end{array}$ & $\begin{array}{l}\text { Retrospective cohort comparing cardiac rehabilitation with usual care with a follow up of } 3000 \text { days. Not a clinical trial. } \\
\text { Main ID: JPRN-UMIN000028237 }\end{array}$ \\
\hline $\begin{array}{l}\text { Hayashi et al. } \\
\qquad(2016)^{33}\end{array}$ & Case-control study. Patients were allocated to fit or unfit groups according to physical capacity. \\
\hline $\begin{array}{l}\text { Bailey et al. } \\
(2018)^{34}\end{array}$ & $\begin{array}{l}\text { RCT evaluating effect of acute exercise on endothelial function in patients with abdominal aortic aneurysm. The study } \\
\text { describes acute flow-mediated-dilatation and not the outcomes of the exercise over a long period as a necessity of treatment } \\
\text { for the disease, so it was considered physical activity and not physical exercises. }\end{array}$ \\
\hline $\begin{array}{l}\text { Weston et al. } \\
\qquad(2017)^{35}\end{array}$ & $\begin{array}{l}\text { RCT assessing the accuracy of high-intensity interval training (HIT) in patients awaiting repair of large abdominal aortic } \\
\text { aneurysms. }\end{array}$ \\
\hline Ongoing studies: & Characteristics: \\
\hline $\begin{array}{l}\text { ClinicalTrials.gov } \\
(2017)^{36}\end{array}$ & $\begin{array}{l}\text { As contacted by e-mail: NCT01805973 (14) has been changed to "The AAA Get fit trial": } \\
\text { Randomized, parallel, blinded to assessors study: } \\
\text { *“...to explore the effectiveness of a 20-week community (either home or gym-based) exercise programme to achieve sustained } \\
\text { improvements in peak VO2 and AT, as measured by CPET, in AAA patients. Changes in QoL, habitual activity levels and } \\
\text { cardiovascular risk will also be assessed." } \\
\text { ClinicalTrials.gov Identifier: NCT02997618 }\end{array}$ \\
\hline $\begin{array}{c}\text { ClinicalTrials.gov } \\
(2017)^{37}\end{array}$ & $\begin{array}{l}\text { Randomized, parallel, open study: † "...to establish if it is possible for patients who have undergone major body surgery to } \\
\text { complete a home based exercise training program and complete the assessments required to measure physical and cognitive } \\
\text { function ... [and] ... whether it is possible to improve the physical function of older patients undergoing major abdominal surgery } \\
\text { in the period following surgery by using a simple exercise regimen that can be carried out at home.." } \\
\text { ClinicalTrials.gov Identifier: NCT03064308 }\end{array}$ \\
\hline $\begin{array}{c}\text { ClinicalTrials.gov } \\
(2017)^{38}\end{array}$ & $\begin{array}{l}\text { Randomized, parallel, blinded to assessors study: } \neq^{\prime \prime . . . c o m p a r i n g ~ t h e ~ e f f e c t ~ o f ~ a ~ " p r e h a b i l i t a t i o n " ~ p r o g r a m ~ t o ~ u s u a l ~ c a r e ~ o n ~} \\
\text { quality of life and clinical outcomes in patients undergoing elective repair of their thoracic aorta." } \\
\text { ClinicalTrials.gov Identifier: NCT02767518 }\end{array}$ \\
\hline Mosk $2017^{39}$ & $\begin{array}{l}\text { Non-randomized, two or more arms study: } \\
\text { II "to evaluate if Multicomponent prehabilitation will reduce postoperative adverse events, primary delirium, which will result in } \\
\text { less long-term adverse consequences." } \\
\text { Main ID: NTR5932 }\end{array}$ \\
\hline \multicolumn{2}{|c|}{ 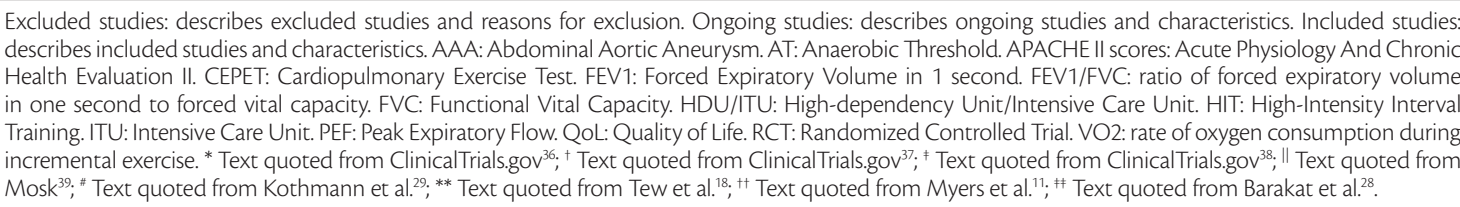 } \\
\hline
\end{tabular}

\section{Risk of bias in included studies}

The Cochrane risk of bias table was used as follows:

Random sequence generation (selection bias): Kothmann et al., ${ }^{29}$ Barakat et al., ${ }^{28}$ and Wnuk et al. ${ }^{30}$ described their randomization methods and were classified as "low risk." The remaining studies did not describe their randomization methods and were classified as "unclear risk." 11,18

Allocation concealment (performance bias and detection bias): all studies were judged to have an "unclear risk" of bias because the allocation method was not described. ${ }^{11,18,28-30}$

Blinding of personnel and participants (performance bias): Due to the nature of the intervention, it was presumably impossible to blind participants and personnel. Therefore, all studies were classified as "high risk" for this domain.

Blinding of outcome assessment (detection bias): Myers et al. ${ }^{11}$ described blinding of the outcome assessment, but the assessors who performed the blinding were not described. Tew et al. ${ }^{18}$ described their study as an open study. Thus, these two studies were judged as "unclear risk." Kothmann et al. ${ }^{29}$ and Wnuk et al. ${ }^{30}$ responded by email regarding the blinding of the outcome assessment and were judged as "low risk." Barakat et al. ${ }^{28}$ described blinding of the outcome assessment and was also judged as "low risk."

Incomplete outcome data (attrition bias): Kothmann et al., ${ }^{29}$ Tew et al., ${ }^{18}$ and Wnuk et al. ${ }^{30}$ described $>20.00 \%$ to $27.41 \%$ of losses to follow-up and the reasons for these losses. How this could impact the results was not clear; therefore, they were graded as "unclear risk." Myers et al. ${ }^{11}$ was graded as "high risk" because the reasons for the $54 \%$ loss to follow-up were uncertain. Barakat et al. ${ }^{28}$ described no loss to follow-up for the proposed outcomes, and their study was judged "low risk."

Selective reporting (reporting bias): All studies described every proposed outcome and were therefore considered to have a "low risk" of bias.

Other potential sources of bias: Myers et al. ${ }^{11}$ described a baseline imbalance between the groups with respect to body mass index $(\mathrm{p}=0.002)$ and the prevalence of diabetes $(30 \%$ in the exercise group vs. $12 \%$ in the usual care group $[\mathrm{p}=0.01])$. To what extent these imbalances could affect the results remained unclear. 
Tew et al. ${ }^{18}$ and Kothmann et al. ${ }^{29}$ did not describe the balance between the intervention and control groups because their study included no $p$ values. These studies were classified as "unclear risk." Barakat et al. ${ }^{28}$ and Wnuk et al. ${ }^{30}$ reported no baseline imbalances. Barakat et al..$^{28}$ used two interventions with different prognoses (endovascular and open surgery), but the number of interventions was balanced between the groups. Therefore, their study was judged "low risk." There was no other source of bias detected in the study by Wnuk et al. ${ }^{30}$; therefore, the study was also judged "low risk."

The authors were contacted, and Kothmann et al. ${ }^{29}$ replied that it is "totally inappropriate to conduct significance tests on baseline values, " citing Senn. ${ }^{40}$ Additionally, these authors did not provide a significance test for the baseline values. This systematic review follows the Cochrane Handbook for Systematic Reviews of Interventions, which recommends inclusion of imbalances between groups in the domain "other source of bias." ${ }^{19}$ The reasons for each judgment are presented in Table 8 .

\section{Effects of interventions}

The following comparisons were analyzed:
Comparison 1: Exercise for patients with small aneurysms during surveillance. ${ }^{11,18,29}$

In total, 106 subjects in the exercise group underwent a 7-week ${ }^{29}$ to 36 -month ${ }^{11}$ supervised exercise program, and 92 were included in no exercise groups.

Proposed outcomes:

- No mortality was reported;

- No patients developed aneurysm rupture;

- The aneurysm growth rate did not change in the pooled studies from the 12-week to 12-month follow-up (mean difference [MD], $-0.05 ; 95 \%$ confidence interval $[\mathrm{CI}],-0.13$ to 0.03$){ }^{11,18}$ Additionally, there was a statistical tendency to reach significance at the $95 \% \mathrm{CI}$;

- Despite the fact that Tew et al..$^{18}$ described quality of life, no data were depicted. The study reported a non-significant change in eight evaluated domains;

- There was a tendency for the number of patients referred for surgery to reduce, but it was not statistically significant (risk ratio [RR], 0.31; 95\% CI, 0.09-1.11) (Figure 2); ${ }^{11,18,29}$

Table 8. Risk of bias table with justifications.

\begin{tabular}{l} 
A) Clinical trials: \\
\hline \multicolumn{1}{c}{ Study/bias } \\
\hline Barakat et al. ${ }^{28}$ \\
Random sequence generation \\
(selection bias) \\
"Low risk"
\end{tabular}

"Low risk"

\begin{abstract}
Quote: "Randomization was performed using opaque, sealed, identical envelopes containing the treatment allocation, according to a computer-generated sequence prepared by an independent professional. Patients were randomized into one of the 2 groups-the exercise (intervention) group or the standard treatment (control) group. The randomization process was witnessed by an independent research professional and was carried out during the initial visit after obtaining informed consent, but before preoperative assessments and interventions." ${ }^{128}$ (p. 48). Comment: randomization was described and seems to be appropriate.
\end{abstract}

Allocation concealment (selection bias) "Unclear risk"

Blinding of participants and personnel (performance bias) - all-cause mortality

"High risk"

Blinding of participants and personnel (performance bias) - number of patients with aortic rupture.

"High risk"

Blinding of participants and personnel (performance bias) - aneurysm growth "High risk"

Blinding of participants and personnel (performance bias) - quality of life
Comment: not described

Quote: "Clinicians including consultant surgeons, anesthetists, department's medical and nursing staff, and interventional radiologists were blinded to patient group allocation. This was ensured by explaining the importance of blinding to all study participants and performing all study procedures in the separate Academic department."28 (p. 48).

Comment: due to the nature of the intervention, it is impossible to blind patients.

Quote: “Clinicians including consultant surgeons, anesthetists, department's medical and nursing staff, and interventional radiologists were blinded to patient group allocation. This was ensured by explaining the importance of blinding to all study participants and performing all study procedures in the separate Academic department."28 (p. 48).

Comment: due to the nature of the intervention it is impossible to blind patients.

Quote: "Clinicians including consultant surgeons, anesthetists, department's medical and nursing staff, and interventional radiologists were blinded to patient group allocation. This was ensured by explaining the importance of blinding to all study participants and performing all study procedures in the separate Academic department."28 (p. 48).

Comment: due to the nature of the intervention it is impossible to blind patients.

Not assessed

Not assessed: The reduction in bias is possible since the authors report the necessary information to avoid bias but it was not described in the study; Not applicable: not possible within the study protocol; Not stated: not described. 
Table 8. Continued...

\section{A) Clinical trials:

Study/bias
Blinding of participants and personnel
(performance bias) - number of
patients referred for surgery
Blinding of participants and personnel
(performance bias) - peri-operative
complications
"High risk" \\ Há Blinding of participants and personnel (performance bias) - postoperative complications "High risk"}

Blinding of participants and personnel (performance bias) - cardiovascular mortality

"High risk"

Blinding of participants and personnel (performance bias) - hospital stay "High risk"

Blinding of participants and personnel (performance bias) - VEF1

Blinding of outcome assessment (detection bias) - all-cause mortality "Low risk"

Blinding of outcome assessment (detection bias) - number of patients with aortic rupture

"Low risk"

Blinding of outcome assessment (detection bias) - aneurysm growth "Low risk"

Blinding of outcome assessment (detection bias) - quality of life Blinding of outcome assessment (detection bias) - number of patients referred for surgery

Blinding of outcome assessment (detection bias) - peri-operative complications

"Low risk"

Blinding of outcome assessment (detection bias) - postoperative complications

"Low risk"

\section{Support for judgment}

Not assessed

Quote: “Clinicians including consultant surgeons, anesthetists, department's medical and nursing staff, and interventional radiologists were blinded to patient group allocation. This was ensured by explaining the importance of blinding to all study participants and performing all study procedures in the separate Academic department."28 (p. 48).

Comment: due to the nature of the intervention it is impossible to blind patients.

Quote: "Clinicians including consultant surgeons, anesthetists, department's medical and nursing staff, and interventional radiologists were blinded to patient group allocation. This was ensured by explaining the importance of blinding to all study participants and performing all study procedures in the separate Academic department." ${ }^{28}$ (p. 48).

Comment: due to the nature of the intervention it is impossible to blind patients.

Quote: "Clinicians including consultant surgeons, anesthetists, department's medical and nursing staff, and interventional radiologists were blinded to patient group allocation. This was ensured by explaining the importance of blinding to all study participants and performing all study procedures in the separate Academic department."28 (p. 48).

Comment: due to the nature of the intervention it is impossible to blind patients.

Quote: "Clinicians including consultant surgeons, anesthetists, department's medical and nursing staff, and interventional radiologists were blinded to patient group allocation. This was ensured by explaining the importance of blinding to all study participants and performing all study procedures in the separate Academic department." ${ }^{28}$ (p. 48).

Comment: due to the nature of the intervention it is impossible to blind patients.

Not assessed

Quote: “Clinicians including consultant surgeons, anesthetists, department's medical and nursing staff, and interventional radiologists were blinded to patient group allocation. This was ensured by explaining the importance of blinding to all study participants and performing all study procedures in the separate Academic department."28 (p. 48).

Comment: blinding of outcome assessment was described and seems to be appropriate

Quote: “Clinicians including consultant surgeons, anesthetists, department's medical and nursing staff, and interventional radiologists were blinded to patient group allocation. This was ensured by explaining the importance of blinding to all study participants and performing all study procedures in the separate Academic department." ${ }^{\prime 2}$ (p. 48).

Comment: blinding of outcome assessment was described and seems to be appropriate

Quote: "Clinicians including consultant surgeons, anesthetists, department's medical and nursing staff, and interventional radiologists were blinded to patient group allocation. This was ensured by explaining the importance of blinding to all study participants and performing all study procedures in the separate Academic department." ${ }^{\prime 28}$ (p. 48).

Comment: blinding of outcome assessment was described and seems to be appropriate Not assessed

Not applicable

Quote: "Clinicians including consultant surgeons, anesthetists, department's medical and nursing staff, and interventional radiologists were blinded to patient group allocation. This was ensured by explaining the importance of blinding to all study participants and performing all study procedures in the separate Academic department."28 (p. 48).

Comment: blinding of outcome assessment was described and seems to be appropriate

Quote: "Clinicians including consultant surgeons, anesthetists, department's medical and nursing staff, and interventional radiologists were blinded to patient group allocation. This was ensured by explaining the importance of blinding to all study participants and performing all study procedures in the separate Academic department."28 (p. 48).

Comment: blinding of outcome assessment was described and seems to be appropriate

Not assessed: The reduction in bias is possible since the authors report the necessary information to avoid bias but it was not described in the study; Not applicable: not possible within the study protocol; Not stated: not described. 
Table 8. Continued...

\begin{tabular}{l} 
A) Clinical trials: \\
\hline Study/bias \\
Blinding of outcome assessment \\
(detection bias) - cardiovascular \\
mortality \\
"Low risk" \\
Blinding of outcome assessment \\
(detection bias) - hospital stay \\
"Low risk" \\
Blinding of outcome assessment \\
(detection bias) - VEF1 \\
Incomplete outcome data (attrition \\
bias) - all-cause mortality \\
"Low risk"
\end{tabular}

Incomplete outcome data (attrition bias) - number of patients with aortic rupture

"Low risk"

Incomplete outcome data (attrition bias) - aneurysm growth

"Low risk"

Incomplete outcome data (attrition bias) - quality of life

Incomplete outcome data (attrition bias) - number of patients referred for surgery

Incomplete outcome data (attrition bias) - peri-operative complications "Low risk"

Incomplete outcome data (attrition bias) - postoperative complications "Low risk"

Incomplete outcome data (attrition bias) - cardiovascular mortality "Low risk"

Incomplete outcome data (attrition bias) - hospital stay

"Low risk"

Incomplete outcome data (attrition bias) - VEF1

Selective reporting (reporting bias) "Low risk"
Support for judgment

Quote: "Clinicians including consultant surgeons, anesthetists, department's medical and nursing staff, and interventional radiologists were blinded to patient group allocation. This was ensured by explaining the importance of blinding to all study participants and performing all study procedures in the separate Academic department."28 (p. 48).

Comment: blinding of outcome assessment was described and seems to be appropriate Quote: "Clinicians including consultant surgeons, anesthetists, department's medical and nursing staff, and interventional radiologists were blinded to patient group allocation. This was ensured by explaining the importance of blinding to all study participants and performing all study procedures in the separate Academic department."28 (p. 48).

Comment: blinding of outcome assessment was described and seems to be appropriate

Not assessed

Quote: "Twelve patients-6 from each group-withdrew from the study before operative interventions as their procedures were cancelled or postponed. No patients were lost to follow-up. Sixty-two patients from each group were included in the final analysis." 28

Comment: there were $8.8 \%$ losses from each group and they were explained. There were no other loses of follow up.

Quote: "Twelve patients-6 from each group - withdrew from the study before operative interventions as their procedures were cancelled or postponed. No patients were lost to follow-up. Sixty-two patients from each group were included in the final analysis." 28

Comment: there were $8.8 \%$ losses from each group and they were explained There were no other loses of follow up.

Quote: "Twelve patients-6 from each group-withdrew from the study before operative interventions as their procedures were cancelled or postponed. No patients were lost to follow-up. Sixty-two patients from each group were included in the final analysis." 28

Comment: there were $8.8 \%$ losses from each group and they were explained There were no other loses of follow up.

Not assessed

Not applicable

Quote: "Twelve patients-6 from each group - withdrew from the study before operative interventions as their procedures were cancelled or postponed. No patients were lost to follow-up. Sixty-two patients from each group were included in the final analysis." 28

Comment: there were $8.8 \%$ losses from each group and they were explained There were no other loses of follow up.

Quote: "Twelve patients-6 from each group - withdrew from the study before operative interventions as their procedures were cancelled or postponed. No patients were lost to follow-up. Sixty-two patients from each group were included in the final analysis." 28

Comment: there were $8.8 \%$ losses from each group and they were explained There were no other loses of follow up.

Quote: "Twelve patients-6 from each group-withdrew from the study before operative interventions as their procedures were cancelled or postponed. No patients were lost to follow-up. Sixty-two patients from each group were included in the final analysis." 28

Comment: there were $8.8 \%$ losses from each group and they were explained There were no other loses of follow up.

Quote: "Twelve patients-6 from each group-withdrew from the study before operative interventions as their procedures were cancelled or postponed. No patients were lost to follow-up. Sixty-two patients from each group were included in the final analysis." 28

Comment: there were $8.8 \%$ losses from each group and they were explained There were no other loses of follow up.

Not assessed

Comment: A protocol has been published: NCT01062594. All proposed outcomes were reported.

Not assessed: The reduction in bias is possible since the authors report the necessary information to avoid bias but it was not described in the study; Not applicable: not possible within the study protocol; Not stated: not described. 
Table 8. Continued...

\begin{tabular}{l} 
A) Clinical trials: \\
\hline \multicolumn{1}{c}{ Study/bias } \\
\hline Other bias \\
"Low risk" \\
Kothmann et al. ${ }^{29}$ \\
Random sequence generation \\
(selection bias) \\
"Low risk" \\
Allocation concealment (selection bias) \\
"Unclear risk" \\
Blinding of participants and personnel \\
(performance bias) - all-cause mortality \\
"High risk"
\end{tabular}

Blinding of participants and personnel (performance bias) - number of patients with aortic rupture.

"High risk"

Blinding of participants and personnel (performance bias) - aneurysm growth Blinding of participants and personnel (performance bias) - quality of life

Blinding of participants and personnel (performance bias) - number of patients referred for surgery "High risk"

Blinding of participants and personnel (performance bias) - peri-operative complications

Blinding of participants and personnel (performance bias) - postoperative complications

Blinding of participants and personnel (performance bias) - cardiovascular mortality

"High risk"

Blinding of participants and personnel (performance bias) - hospital stay

Blinding of participants and personnel (performance bias) - VEF1

Blinding of outcome assessment (detection bias) - all-cause mortality "Low risk"

Blinding of outcome assessment (detection bias) - number of patients with aortic rupture

"Low risk"

Blinding of outcome assessment (detection bias) - aneurysm growth Blinding of outcome assessment (detection bias) - quality of life Blinding of outcome assessment (detection bias) - number of patients referred for surgery

"Low risk"

\section{Support for judgment}

Comment: there were no imbalances between groups. There were two different interventions (endovascular and open surgery). The numbers were balanced between groups.

Quote: “...participants were randomly allocated (via sealed envelopes) to a supervised exercise intervention or to the control group (usual care)." 29

Comment: Randomization was described and seems to be appropriate.

Comment: not described

Comment: no information provided. Probably not done due to the nature of the intervention.

Comment: no information provided. Probably not done due to the nature of the intervention.

Not assessed

Not assessed

Comment: no information provided. Probably not done due to the nature of the intervention.

Not applicable

Not applicable

Comment: no information provided. Probably not done due to the nature of the intervention.

Not applicable

Not assessed

Quote: "In the paper we state: "The investigator reading AT results (G.D.) was blinded to group allocation." Simply, GD was provided with the output from the cardiopulmonary exercise tests and derived the anaerobic threshold for each participant without knowledge of group assignment, i.e., blind."29 Provided by email

Comment: blinding of outcome assessment properly described.

Quote: "In the paper we state: "The investigator reading AT results (G.D.) was blinded to group allocation." Simply, GD was provided with the output from the cardiopulmonary exercise tests and derived the anaerobic threshold for each participant without knowledge of group assignment, i.e., blind."29 Provided by email

Comment: blinding of outcome assessment properly described.

Not assessed

Not assessed

Quote: "In the paper we state: "The investigator reading AT results (G.D.) was blinded to group allocation." Simply, GD was provided with the output from the cardiopulmonary exercise tests and derived the anaerobic threshold for each participant without knowledge of group assignment, i.e., blind."29 Provided by email

Comment: blinding of outcome assessment properly described.

Not assessed: The reduction in bias is possible since the authors report the necessary information to avoid bias but it was not described in the study; Not applicable: not possible within the study protocol; Not stated: not described. 
Table 8. Continued...

A) Clinical trials:

\begin{tabular}{l} 
Study/bias \\
\hline Blinding of outcome assessment \\
(detection bias) - peri-operative \\
complications \\
Blinding of outcome assessment \\
(detection bias) - postoperative \\
complications \\
Blinding of outcome assessment \\
(detection bias) - cardiovascular \\
mortality \\
"Low risk" \\
Blinding of outcome assessment \\
(detection bias) - hospital stay \\
Blinding of outcome assessment \\
(detection bias) - VEF1 \\
Incomplete outcome data (attrition \\
bias) - all-cause mortality \\
"Unclear risk"
\end{tabular}

Incomplete outcome data (attrition bias) - number of patients with aortic rupture

"Unclear risk"

Incomplete outcome data (attrition bias) - aneurysm growth

Incomplete outcome data (attrition bias) - quality of life Incomplete outcome data (attrition bias) - number of patients referred for surgery

"unclear risk"

Incomplete outcome data (attrition bias) - peri-operative complications Incomplete outcome data (attrition bias) - postoperative complications Incomplete outcome data (attrition bias) - cardiovascular mortality "Unclear risk"

Incomplete outcome data (attrition bias) - hospital stay

Incomplete outcome data (attrition bias) - VEF1

Selective reporting (reporting bias) "Low risk"

Other bias

"Unclear risk"

Myers et al. ${ }^{11}$

Random sequence generation (selection bias)

"Unclear risk"
Support for judgment

Not applicable

Not applicable

Quote: "In the paper we state: "The investigator reading AT results (G.D.) was blinded to group allocation." Simply, GD was provided with the output from the cardiopulmonary exercise tests and derived the anaerobic threshold for each participant without knowledge of group assignment, i.e., blind." 29 Provided by email

Comment: blinding of outcome assessment properly described.

Not applicable

Not assessed

"Of these, 17 of 20 and eight of 10 completed the study period in the exercise and control groups, respectively, producing full data sets for analysis" 29

Comment: (There was $15 \%$ losses from the intervention group and $20 \%$ from the control group. We are not sure about the extent to which this could affect the results).

"Of these, 17 of 20 and eight of 10 completed the study period in the exercise and control groups, respectively, producing full data sets for analysis" 29

Comment: (There was $15 \%$ losses from the intervention group and $20 \%$ from the control group. We are not sure about the extent to which this could affect the results).

Not assessed

Not assessed

"Of these, 17 of 20 and eight of 10 completed the study period in the exercise and control groups, respectively, producing full data sets for analysis" 29

Comment: There was $15 \%$ losses from the intervention group and $20 \%$ from the control group. We are not sure to what extent this could affect the results.

Not applicable

Not applicable

"Of these, 17 of 20 and eight of 10 completed the study period in the exercise and control groups, respectively, producing full data sets for analysis" 29

Comment: There was $15 \%$ losses from the intervention group and $20 \%$ from the control group. We are not sure to what extent this could affect the results.

Not applicable

Not assessed

Comment: All the proposed outcomes were reported.

Comment: There is an uncertainty about the balance between groups at baseline, since no $\mathrm{p}$ value was provided. We are not sure to what extent this could affect the results.

Quote: "One hundred and forty patients with small AAAs (72 T $8 \mathrm{yr}$ ) were randomised to exercise training $(n=72)$ or usual care $(n=68)^{\prime \prime 11}$

Comment: Not described.

Allocation concealment (selection bias) Comment: not described "Unclear risk"

Not assessed: The reduction in bias is possible since the authors report the necessary information to avoid bias but it was not described in the study; Not applicable: not possible within the study protocol; Not stated: not described. 
Table 8. Continued...

\section{A) Clinical trials:}

\section{Study/bias}

Blinding of participants and personnel (performance bias) - all-cause mortality

"High risk"

Blinding of participants and personnel (performance bias) - number of patients with aortic rupture.

"High risk"

Blinding of participants and personnel (performance bias) - aneurysm growth "High risk"

Blinding of participants and personnel (performance bias) - quality of life

Blinding of participants and personnel (performance bias) - number of patients referred for surgery

"High risk"

Blinding of participants and personnel (performance bias) - peri-operative complications

Blinding of participants and personnel (performance bias) - postoperative complications

Blinding of participants and personnel (performance bias) - cardiovascular mortality

"High risk"

Blinding of participants and personnel (performance bias) - hospital stay

Blinding of participants and personne (performance bias) - VEF1

Blinding of outcome assessment (detection bias) - all-cause mortality "Unclear risk"

Blinding of outcome assessment (detection bias) - number of patients with aortic rupture

"Unclear risk"

Blinding of outcome assessment (detection bias) - aneurysm growth "Unclear risk"

Blinding of outcome assessment (detection bias) - quality of life

Blinding of outcome assessment (detection bias) - number of patients referred for surgery

"Unclear risk"

Blinding of outcome assessment (detection bias) - peri-operative complications

Blinding of outcome assessment (detection bias) - postoperative complications
Support for judgment

Comment: not stated. Probably not done since the nature of intervention precluded this masking

Comment: not stated. Probably not done since the nature of intervention precluded this masking

Comment: not stated. Probably not done since the nature of intervention precluded this masking

Not assessed

Comment: not stated. Probably not done since the nature of intervention precluded this masking

Not assessed

Not assessed

Comment: not stated. Probably not done since the nature of intervention precluded this masking

Not assessed

Not assessed

Quote: "Both the RVT and the individual making the diameter measurements were blinded to group randomisation". Answered by e-mail: "both were unaware of the intervention or control group."11

Comment: no information provided for data assessors

Quote: Quote: "Both the RVT and the individual making the diameter measurements were blinded to group randomisation". Answered by e-mail: "both were unaware of the intervention or control group."11

Comment: no information provided for data assessors

Quote: "Both the RVT and the individual making the diameter measurements were blinded to group randomisation". Answered by e-mail: "both were unaware of the intervention or control group."11

Comment: no information provided for data assessors

Not assessed

Quote: "Both the RVT and the individual making the diameter measurements were blinded to group randomisation". Answered by e-mail: "both were unaware of the intervention or control group."11

Comment: no information provided for data assessors

Not assessed

Not assessed

Not assessed: The reduction in bias is possible since the authors report the necessary information to avoid bias but it was not described in the study; Not applicable: not possible within the study protocol; Not stated: not described. 
Table 8. Continued...

\begin{tabular}{l} 
A) Clinical trials: \\
\hline Study/bias \\
\hline Blinding of outcome assessment \\
(detection bias) - cardiovascular \\
mortality \\
"Unclear risk" \\
Blinding of outcome assessment \\
(detection bias) - hospital stay \\
Blinding of outcome assessment \\
(detection bias) - VEF1 \\
Incomplete outcome data (attrition \\
bias) - all-cause mortality \\
"High risk"
\end{tabular}

Incomplete outcome data (attrition bias) - number of patients with aortic rupture

"High risk"

Incomplete outcome data (attrition bias) - aneurysm growth

"High risk"

Incomplete outcome data (attrition bias) - quality of life

Incomplete outcome data (attrition bias) - number of patients referred for surgery

"High risk"

Incomplete outcome data (attrition bias) - peri-operative complications Incomplete outcome data (attrition bias) - postoperative complications Incomplete outcome data (attrition bias) - cardiovascular mortality "High risk"

Incomplete outcome data (attrition bias) - hospital stay

Incomplete outcome data (attrition bias) - VEF1

Selective reporting (reporting bias) "Low risk"

Other bias

"Unclear risk"

Tew et al. ${ }^{18}$

Random sequence generation

(selection bias)

"Unclear risk"

Allocation concealment (selection bias) "Unclear risk"

Blinding of participants and personnel (performance bias) - all-cause mortality

"High risk"

Not assessed: The reduction in bias is possible since the authors report the necessary information to avoid bias but it was not described in the study; Not applicable: not possible within the study protocol; Not stated: not described.
Quote: "Both the RVT and the individual making the diameter measurements were blinded to group randomisation". Answered by e-mail: "both were unaware of the intervention or control group." 11

Comment: no information provided for data assessors

Not assessed

Not assessed

Quote: "81\% of subjects completed at least 1 year in the trial"11

Comment: There were $19 \%$ losses in one year. Additionally, at the end of follow-up, there were 39 losses from the intervention group and 36 from the control group. The reasons for these losses are unclear.

Quote: "81\% of subjects completed at least 1 year in the trial"11

Comment: There were $19 \%$ losses in one year. Additionally, at the end of follow-up, there were 39 losses from the intervention group and 36 from the control group. The reasons for these losses are unclear.

Quote: "81\% of subjects completed at least 1 year in the trial"11

Comment: There were $19 \%$ losses in one year. Additionally, at the end of follow-up, there were losses are unclear.

Not assessed

Quote: " $81 \%$ of subjects completed at least 1 year in the trial"11

Comment: There were $19 \%$ losses in one year. Additionally, at the end of follow-up, there were 39 losses from the intervention group and 36 from the control group. The reasons for these losses are unclear.

Not assessed

\section{Not assessed}

Quote: "81\% of subjects completed at least 1 year in the trial"11

Comment: There were $19 \%$ losses in one year. Additionally, at the end of follow-up, there were 39 losses from the intervention group and 36 from the control group. The reasons for these losses are unclear.

Not assessed

Not assessed

Quote: Protocol available at Clinicaltrials.gov, identifier: NCT00349947.

Comment: protocol described. All proposed outcomes were reported

Comment: There was an "imbalance between groups at baseline regarding BMI mean at baseline ( $p=0.002)$ and frequency of diabetes (30\% vs. $12 \%$ in the exercise and usual care groups, respectively, $P=0.01) . " 11$

We are not sure to what extent this could affect the results.

Quote: "Allocation to exercise or control was done using a randomization sequence created by an independent researcher before study commencement." ${ }^{\prime 18}$

Comment: unclear information

Quote: "The study researchers were made aware of this sequence on a case-by-case basis after baseline assessments were completed."18

Comment: unclear information

Quote: "Ventilatory threshold was determined by an independent exercise physiologist blinded to group allocation using the $v$-slope and ventilatory equivalents methods." ${ }^{\prime 18}$ Comment: The nature of the intervention precluded this masking 39 losses from the intervention group and 36 from the control group. The reasons for these 
Table 8. Continued...

A) Clinical trials:

\section{Study/bias}

Blinding of participants and personnel (performance bias) - number of patients with aortic rupture.

"High risk"

Blinding of participants and personnel (performance bias) - aneurysm growth "High risk"

Blinding of participants and personnel (performance bias) - quality of life "High risk"

Blinding of participants and personnel (performance bias) - number of patients referred for surgery

Blinding of participants and personnel (performance bias) - peri-operative complications

Blinding of participants and personnel (performance bias) - postoperative complications

Blinding of participants and personnel (performance bias) - cardiovascular mortality

"High risk"

Blinding of participants and personnel (performance bias) - hospital stay

Blinding of participants and personnel (performance bias) - VEF1

Blinding of outcome assessment (detection bias) - all-cause mortality "High risk"

Blinding of outcome assessment (detection bias) - number of patients with aortic rupture

"High risk"

Blinding of outcome assessment (detection bias) - aneurysm growth "High risk"

Blinding of outcome assessment (detection bias) - quality of life "High risk"

Blinding of outcome assessment (detection bias) - number of patients referred for surgery

Blinding of outcome assessment (detection bias) - peri-operative complications

Blinding of outcome assessment (detection bias) - postoperative complications

Blinding of outcome assessment (detection bias) - cardiovascular mortality

"High risk"

Blinding of outcome assessment (detection bias) - hospital stay
Support for judgment

Quote: "Ventilatory threshold was determined by an independent exercise physiologist blinded to group allocation using the $v$-slope and ventilatory equivalents methods." ${ }^{\prime 18}$

Comment: The nature of the intervention precluded this masking

Quote: "Ventilatory threshold was determined by an independent exercise physiologist blinded to group allocation using the $v$-slope and ventilatory equivalents methods." ${ }^{\prime 18}$

Comment: The nature of the intervention precluded this masking

Quote: "Ventilatory threshold was determined by an independent exercise physiologist blinded to group allocation using the $v$-slope and ventilatory equivalents methods."18

Comment: The nature of the intervention precluded this masking

Not assessed

Not applicable

Not applicable

Quote: "Ventilatory threshold was determined by an independent exercise physiologist blinded to group allocation using the $v$-slope and ventilatory equivalents methods."18

Comment: The nature of the intervention precluded this masking

Not applicable

Not assessed

Quote: "The study researchers were made aware of this sequence on a case-by-case basis after baseline assessments were completed".18

Comment: There was no blinding

Quote: "The study researchers were made aware of this sequence on a case-by-case basis after baseline assessments were completed".18

Comment: There was no blinding

Quote: "The study researchers were made aware of this sequence on a case-by-case basis after baseline assessments were completed". 18

Comment: There was no blinding

Quote: The study researchers were made aware of this sequence on a case-by-case basis after baseline assessments were completed"18

Comment: There was no blinding

Not assessed

Not applicable

Not applicable

Quote: The study researchers were made aware of this sequence on a case-by-case basis after baseline assessments were completed".18

Comment: There was no blinding

Not applicable

Not assessed: The reduction in bias is possible since the authors report the necessary information to avoid bias but it was not described in the study; Not applicable: not possible within the study protocol; Not stated: not described. 
Table 8. Continued...

\begin{tabular}{l} 
A) Clinical trials: \\
\hline Study/bias \\
Blinding of outcome assessment \\
(detection bias) - VEF1 \\
Incomplete outcome data (attrition \\
bias) - all-cause mortality \\
"Unclear risk"
\end{tabular}

Incomplete outcome data (attrition bias) - number of patients with aortic rupture

"Unclear risk"

Incomplete outcome data (attrition bias) - aneurysm growth

"Unclear risk" bias) - quality of life

"Unclear risk"

Incomplete outcome data (attrition bias) - number of patients referred for surgery

Incomplete outcome data (attrition bias) - peri-operative complications Incomplete outcome data (attrition bias) - postoperative complications Incomplete outcome data (attrition bias) - cardiovascular mortality "Unclear risk"

Incomplete outcome data (attrition bias) - hospital stay

Incomplete outcome data (attrition

Selective reporting (reporting bias) "Low risk"

Other bias

"Unclear risk

Wnuk et al. ${ }^{30}$

Random sequence generation (selection bias)

"Low risk"
Incomplete outcome data (attrition bias) - VEF1

\section{Not assessed}

Quote: "Three participants did not complete the exercise intervention: 1 withdrew because of being diagnosed with cancer, 1 underwent pacemaker implantation, and 1 suffered a back injury at home"18

Comment: There were about $20 \%$ of losses from the intervention group, with reasons provided. We are not sure to what extent this could affect the results.

Quote: "Three participants did not complete the exercise intervention: 1 withdrew because of being diagnosed with cancer, 1 underwent pacemaker implantation, and 1 suffered a back injury at home"18

Comment: There were about $20 \%$ of losses from the intervention group, with reasons provided. We are not sure to what extent this could affect the results.

Quote: "Three participants did not complete the exercise intervention: 1 withdrew because of being diagnosed with cancer, 1 underwent pacemaker implantation, and 1 suffered a back injury at home"18

Comment: There were about $20 \%$ of losses from the intervention group, with reasons provided. We are not sure to what extent this could affect the results.

Quote: "Three participants did not complete the exercise intervention: 1 withdrew because of being diagnosed with cancer, 1 underwent pacemaker implantation, and 1 suffered a back injury at home"18

Comment: There were about $20 \%$ of losses from the intervention group, with reasons provided. We are not sure to what extent this could affect the results.

Not assessed

Not applicable

Not applicable

Quote: "Three participants did not complete the exercise intervention: 1 withdrew because of being diagnosed with cancer, 1 underwent pacemaker implantation, and 1 suffered a back injury at home"18

Comment: There were about $20 \%$ of losses from the intervention group, with reasons provided. We are not sure to what extent this could affect the results.

Not applicable

Not assessed

Quote: "The study was registered in ClinicalTrials.gov under reference no. NCT01234610."18 Comment: there is a protocol and it seems to be appropriate. All proposed outcomes were reported.

Does not show the data for the quality of life outcome: “...or any of the 8 quality of life domains (P.05; data not presented)"18

Comment: There is an uncertainty about the balance between groups at baseline, since no $p$ value was provided. We are not sure to what extent this could affect the results.

Quote: "The randomization of the study was conducted by drawing envelopes containing a number of the appropriate group - single blind study. Patients with the number 1 were qualified for the experimental group with backward walking training (group I), with number 2 for the experimental group with forward walking training (group II) and 3 for the control group."30 Comment: randomization considered done and apparently appropriate.

Allocation concealment (selection bias) Comment: not stated

"Unclear risk"

Blinding of participants and personnel Not assessed

(performance bias) - all-cause

mortality

Not assessed: The reduction in bias is possible since the authors report the necessary information to avoid bias but it was not described in the study; Not applicable: not possible within the study protocol; Not stated: not described. 
Table 8. Continued...

A) Clinical trials:

\section{Study/bias}

Blinding of participants and personnel (performance bias) - number of patients with aortic rupture.

Blinding of participants and personnel (performance bias) - aneurysm growth

Blinding of participants and personnel (performance bias) - quality of life

Blinding of participants and personnel Not applicable (performance bias) - number of patients referred for surgery

Blinding of participants and personnel Not applicable (performance bias) - peri-operative complications

Blinding of participants and personnel (performance bias) - postoperative complications

Blinding of participants and personnel (performance bias) - cardiovascular mortality

Blinding of participants and personnel (performance bias) - hospital stay "High risk"

Blinding of participants and personnel (performance bias) - VEF1

"High risk"

Blinding of outcome assessment (detection bias) - all-cause mortality

Blinding of outcome assessment (detection bias) - number of patients with aortic rupture

Blinding of outcome assessment (detection bias) - aneurysm growth

Blinding of outcome assessment (detection bias) - quality of life Blinding of outcome assessment (detection bias) - number of patients referred for surgery

Blinding of outcome assessment (detection bias) - peri-operative complications

Blinding of outcome assessment (detection bias) - postoperative complications

Blinding of outcome assessment (detection bias) - cardiovascular mortality

Blinding of outcome assessment (detection bias) - hospital stay "Low risk"

Blinding of outcome assessment (detection bias) - VEF1

"Low risk"

Not applicable

Not applicable

Not assessed

Not assessed

Not assessed

Not assessed

Not applicable

Not applicable

Not assessed

Not applicable

Not applicable

Not assessed

Not assessed
Support for judgment

Quote: "single blind study"30

Comment: not blinded

Quote: "single blind study"30

Comment: not blinded

Quote: described as a single blinded study. Replied by e-mail 09/27/2017: "Measurement of gait parameters and spirometry was evaluated by physiotherapist from the Department of Rehabilitation. Routine physiotherapy and training walking in three groups was conducted by physiotherapist from the department of General and Vascular Surgery." ${ }^{130}$

Comment: blinding of outcome assessment was described and seems to be appropriate.

Quote: described as a single blinded study. Replied by e-mail 09/27/2017: "Measurement of gait parameters and spirometry was evaluated by physiotherapist from the Department of Rehabilitation. Routine physiotherapy and training walking in three groups was conducted by physiotherapist from the department of General and Vascular Surgery."30

Comment: blinding of outcome assessment was described and seems to be appropriate.

Not assessed: The reduction in bias is possible since the authors report the necessary information to avoid bias but it was not described in the study; Not applicable: not possible within the study protocol; Not stated: not described. 
Table 8. Continued...

\begin{tabular}{|c|c|}
\hline \\
\hline$\frac{\text { A) Clinical trials: }}{\text { Study/bias }}$ & Support for judgment \\
\hline $\begin{array}{l}\text { Incomplete outcome data (attrition } \\
\text { bias) - all-cause mortality }\end{array}$ & Not assessed \\
\hline $\begin{array}{l}\text { Incomplete outcome data (attrition } \\
\text { bias) - number of patients with aortic } \\
\text { rupture }\end{array}$ & Not applicable \\
\hline $\begin{array}{l}\text { Incomplete outcome data (attrition } \\
\text { bias) - aneurysm growth }\end{array}$ & Not applicable \\
\hline $\begin{array}{l}\text { Incomplete outcome data (attrition } \\
\text { bias) - quality of life }\end{array}$ & Not assessed \\
\hline $\begin{array}{l}\text { Incomplete outcome data (attrition } \\
\text { bias) - number of patients referred for } \\
\text { surgery }\end{array}$ & Not applicable \\
\hline $\begin{array}{l}\text { Incomplete outcome data (attrition } \\
\text { bias) - peri-operative complications }\end{array}$ & Not applicable \\
\hline $\begin{array}{l}\text { Incomplete outcome data (attrition } \\
\text { bias) - postoperative complications }\end{array}$ & Not assessed \\
\hline $\begin{array}{l}\text { Incomplete outcome data (attrition } \\
\text { bias) - cardiovascular mortality }\end{array}$ & Not assessed \\
\hline $\begin{array}{l}\text { Incomplete outcome data (attrition } \\
\text { bias) - hospital stay } \\
\text { "Unclear risk" }\end{array}$ & $\begin{array}{l}\text { Comment: there were } 27.41 \% \text { dropouts and we do not what the impact of this would be on } \\
\text { results and conclusions. }\end{array}$ \\
\hline $\begin{array}{l}\text { Incomplete outcome data (attrition } \\
\text { bias) - VEF1 } \\
\text { "Unclear risk" }\end{array}$ & $\begin{array}{l}\text { Comment: there were } 27.41 \% \text { dropouts and we do not what the impact of this would be on } \\
\text { results and conclusions. }\end{array}$ \\
\hline $\begin{array}{l}\text { Selective reporting (reporting bias) } \\
\text { "Low risk" }\end{array}$ & Comment: the proposed outcomes were described and seem to be appropriate. \\
\hline $\begin{array}{l}\text { Other bias } \\
\text { "Low risk" }\end{array}$ & Comment: describes balance between groups at baseline. No other source of bias detected. \\
\hline
\end{tabular}

- Cardiovascular adverse events were not different between the intervention and control groups (RR, 1.57; 95\% CI, 0.07-35.46). ${ }^{11,18,29}$ In the study by Kothmann et al., ${ }^{29}$ one patient in the intervention group had a severe adverse cardiac event after seven sessions.

Non-proposed outcomes:

There was an improvement in the exercise time at 12 weeks (MD, 105.86; 95\% CI, 40.29-171.43) (Figure 3A). ${ }^{11,18}$ This result was even stronger in the 12-month clinical trial (MD, 142.00; 95\% CI, 63.43-220.57) (Figure 3B). ${ }^{11}$

The change in anaerobic threshold improved after at least 7 weeks of exercise (MD, 1.55; 95\% CI, 0.27-2.82) (Figure 4A). ${ }^{11,18,29}$ Although the maximal rate of oxygen consumption during incremental exercise $\left(\mathrm{VO}_{2}\right.$ peak) improved, the difference did not attain statistical significance (MD, $1.15 ; 95 \% \mathrm{CI}$, -0.09 to 2.38 ) (Figure 4B). ${ }^{11,18,29}$

Comparison 2: Exercise in the preoperative period ${ }^{28}$

One study assessed this comparison. ${ }^{28}$ The study included 62 patients in the exercise group and 62 in the no exercise group for 6 weeks. Data were assessed in the interquartile range, which was transformed to standard deviation by dividing the interquartile range by 1.35 , as described in the seventh chapter of the Cochrane handbook. ${ }^{19}$

Proposed outcomes:

- There was no difference in 30-day mortality between the groups (RR, 1.00; 95\% CI, 0.93-1.08) (Figure 5);

- No participants developed an aneurysm rupture;

- Quality of life was not measured;

- Overall, postoperative complications were reduced in the exercise group (RR, 0.54; 95\% CI, 0.31-0.93). In a subgroup analysis, cardiac complications (RR, 0.36; 95\% CI, 0.14-0.93), and renal complications (RR, $0.31 ; 95 \% \mathrm{CI}$, $0.11-0.89)$ had the most important benefit. Despite a tendency to reduce pulmonary complications, this was not statistically significant (RR, 0.54; $95 \%$ CI, 0.23-1.26). When analyzed by surgical 
subgroups, renal complications were lower in open aneurysm surgery (RR, $0.54 ; 95 \%$ CI, 0.34-0.87) than in endovascular repair (RR, 1.00; 95\% CI, 0.07-15.04). The same trend occurred in cardiac complications: open aneurysm repair (RR, 0.36 ; 95\% CI, 0.13-1.04) versus an endovascular approach (RR, 0.33; 95\% CI, 0.04-2.97). Pulmonary complications were not significantly reduced in endovascular repair (RR, $0.11 ; 95 \% \mathrm{CI}, 0.01-1.95$ ) or open repair (RR, 0.78; 95\% CI, 0.32-1.88);

- Hospital stay was not reduced in endovascular repair (MD, $-1.00 ; 95 \% \mathrm{CI},-4.22$ to 2.22 ) or open aneurysm repair groups (MD, $0.00 ; 95 \% \mathrm{CI}$, -0.55 to 0.55 );
- There was a detectable reduction in the critical care stay in the exercise group (MD, -1.00 ; $95 \% \mathrm{CI},-1.26$ to -0.74$)$.

Eleven of 62 patients who were referred for exercise (17.7\%) did not attend the scheduled exercise sessions. There were no losses to follow-up after initiating the study.

Non-proposed outcomes:

Bleeding was described clinically or as a need for transfusion of more than four bags and was not affected by inclusion in either the preoperative exercise or no exercise study groups (RR, $0.57 ; 95 \% \mathrm{CI}, 0.18-1.85){ }^{28}$

There was an improvement in anaerobic threshold (MD, 1.80; 95\% CI, 0.68-2.92) and $\mathrm{VO}_{2}$ peak oxygen consumption (MD, 1.60; 95\% CI, 0.40-2.80). ${ }^{28}$

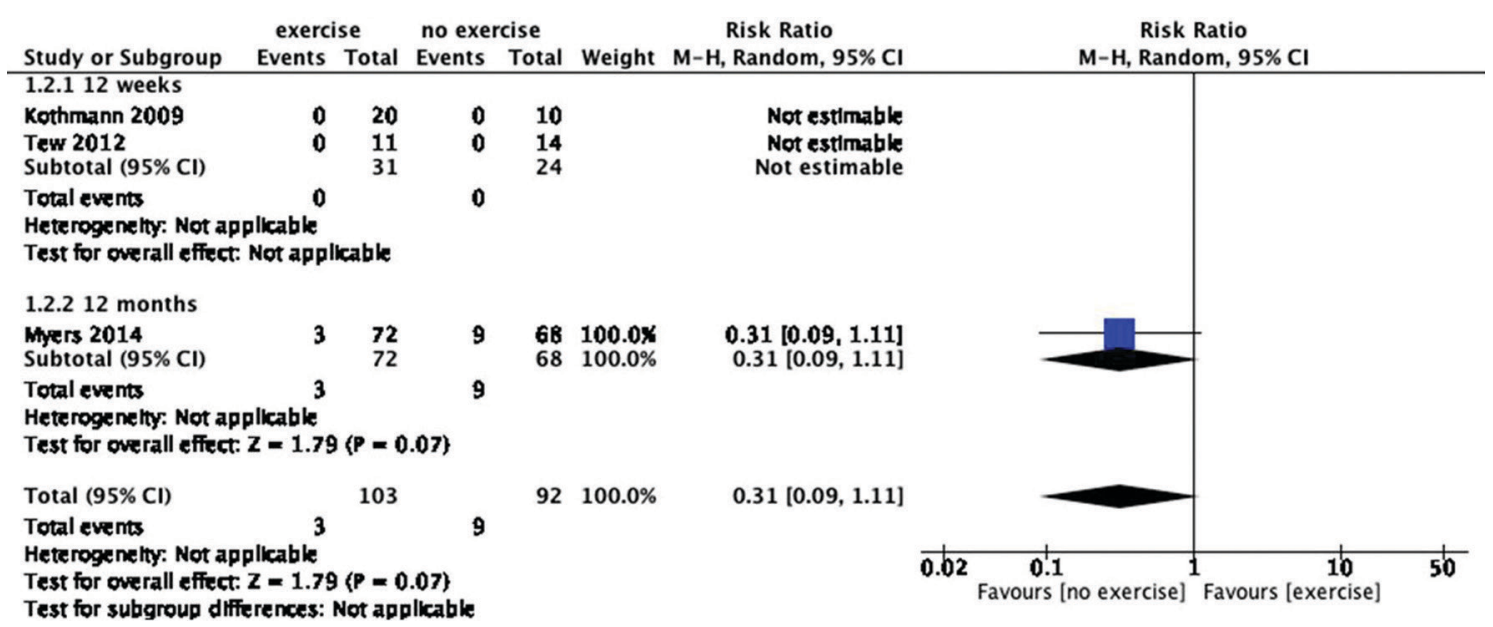

Figure 2. Number of patients referred for surgery at any time during surveillance. This figure shows patients referred for surgery at any time during the surveillance period. They were described at 12 weeks (1.2.1) and at 12 months (1.2.2). M-H, Mantel-Haenszel; Random, random-effects model; $\mathrm{Cl}$, confidence interval; Events, number of patients referred for surgery; Total, total number of patients; Total $(95 \% \mathrm{Cl})$, effect size at $95 \%$ confidence interval.

A

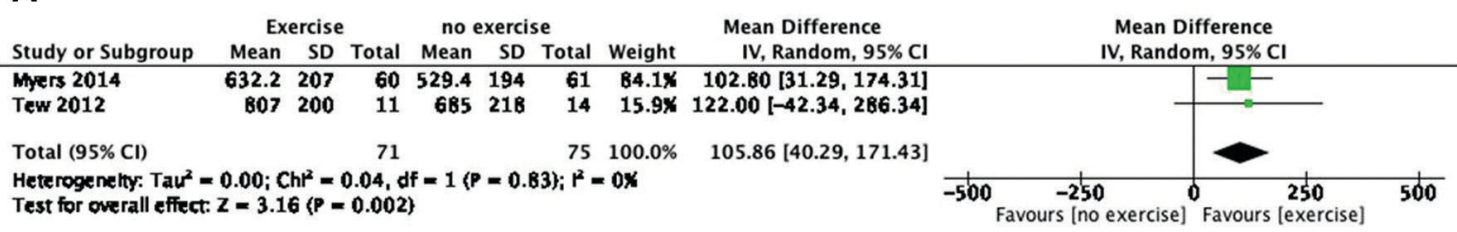

B

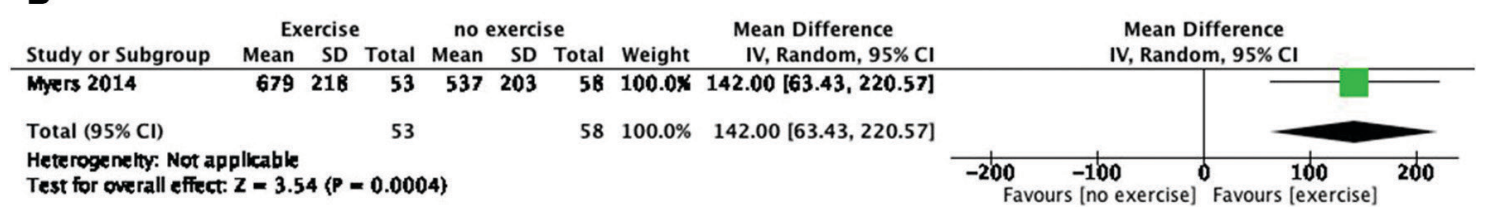

Figure 3. Exercise time during surveillance. (A) Exercise time during surveillance at 12 weeks (exercise vs. no exercise). (B) Exercise time during surveillance at 12 months (exercise vs. no exercise). IV, inverse variance; Random, random-effects model; $\mathrm{Cl}$, confidence interval; Total, total number of patients; Total ( $95 \% \mathrm{Cl}$ ), effect size at $95 \%$ confidence interval. 
Comparison 3: Exercise in the preoperative and postoperative periods $s^{30}$

One of the studies included assessed 22 patients who performed backward walking, 22 who performed forwarding walking, and 21 in a control group during the preoperative and postoperative periods. After contact, the author reported 18 drop-outs: 7 in the backward walking group (due to myocardial infarction in 3 patients, respiratory failure in 3 , and refusal to exercise after surgery in 1), 6 in the forward walking group (myocardial infarction in 2 patients, respiratory failure in 2 , and exclusion due to blood coagulation dysfunction in 2), and 5 patients in the control group (all due to myocardial infarction). A per-protocol analysis was conducted (including 15 patients in the backward walking group, 16 in

A

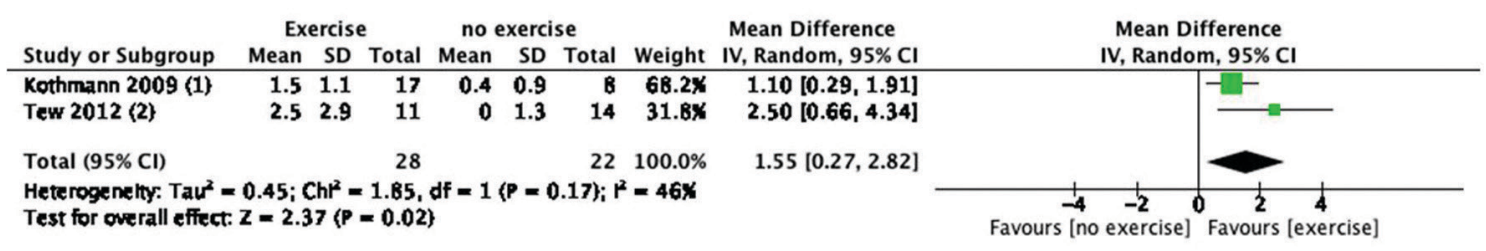

Footnotes

(1) At 7 weeks

(2) at 12 weeks

B

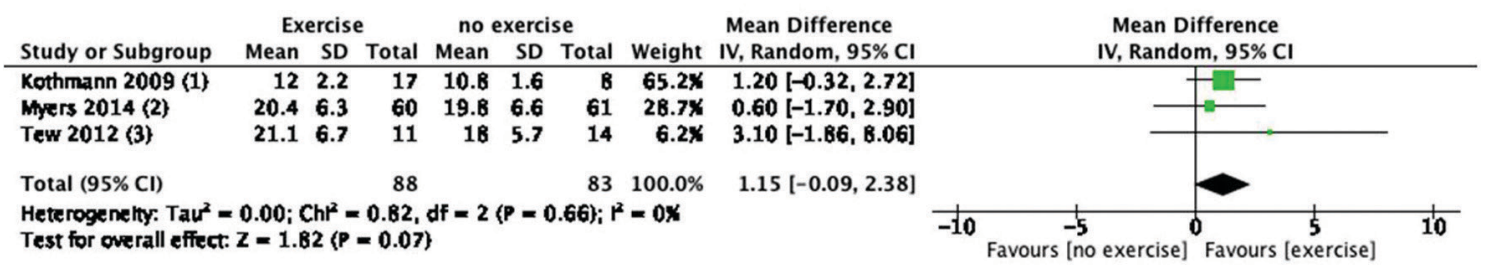

Footnotes

(1) 7 weeks

(2) 12 weeks

(3) 12 weeks

Figure 4. Change in anaerobic threshold and peak $\mathrm{VO}_{2}$ during surveillance. (A) Change in total anaerobic threshold values at 7 and 12 weeks during surveillance (exercise vs. no exercise). (B) Peak $\mathrm{VO}_{2}$ during surveillance at 7 and 12 weeks (exercise vs. no exercise). IV, inverse variance; Random, random-effects model; $\mathrm{Cl}$, confidence interval; Total, total number of patients; Total ( $95 \% \mathrm{Cl}$ ), effect size at 95\% confidence interval.

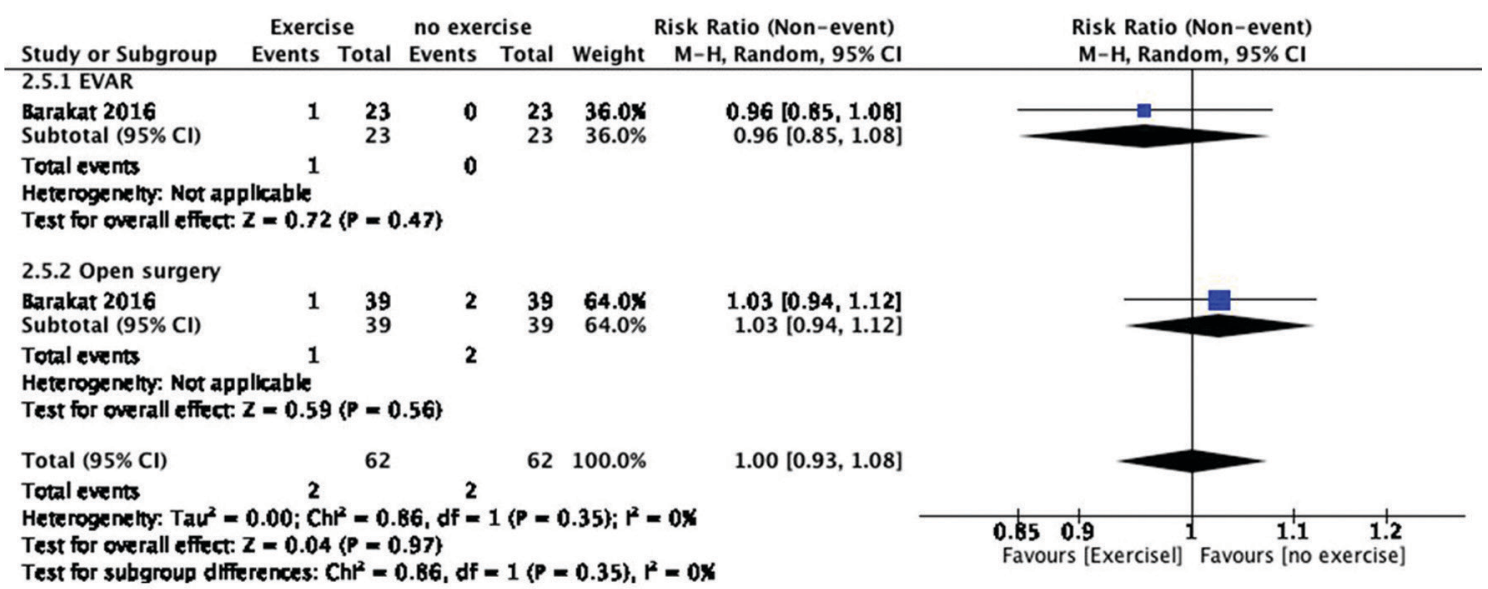

Figure 5. Thirty-day mortality after surgery in patients in the preoperative study. This figure compares 30-day mortality after surgery in patients in the exercise and no exercise groups during the preoperative period. EVAR, endovascular aneurysm repair. Comparison 2 involves the subset treated with EVAR (2.5.1) and the subset treated with open surgery (2.5.2). M-H: Mantel-Haenszel; Random, random-effects model; $\mathrm{Cl}$, confidence interval; Events, number of deaths up to 30 days after surgery; Total, total number of patients; Total $(95 \% \mathrm{Cl})$, effect size at 95\% confidence interval. 
the forwarding walking group, and 16 in the control group). All patients were men, and the results for proposed outcomes were as follows:

- No mortality was reported;

- Quality of life was not measured;

- The number of participants presenting with at least one severe complication was not reported;

- The hospital stay was detectably reduced in the forward walking group compared with the control group (MD, $-0.69 ; 95 \% \mathrm{CI},-1.24$ to -0.14 ). No difference was observed between the backward walking group and control group (MD, -0.06 ; $95 \% \mathrm{CI},-0.53$ to 0.41 );

- Length of intensive care unit stay after aneurysm surgery (in days) was not assessed;

- During forward walking, the forced expiratory volume in 1 second was not different between the intervention group and control group (RR, 0.27; $95 \% \mathrm{CI},-0.12$ to 0.66 ).

The proposed subgroup analysis was not performed because of limited data available.

Using GRADEpro-GDT, ${ }^{24}$ we judged the quality of the evidence as "very low" for all outcomes. Quality ratings were downgraded due to methodological limitations (impossibility of blinding personnel and participants, attrition bias) and imprecision (single study for some outcomes and low numbers of participants) (Tables 2, 3, 4 and 5).

\section{DISCUSSION}

This review revealed no differences in mortality rates between patients with and without exercise during surveillance, preoperative, or postoperative periods. Additionally, no aneurysm ruptures were detected in any intervention groups (total of 209 patients). These clinical trials did not identify reduction in aneurysmal expansion rates or referrals for surgery (Figure 2). However, 6 weeks of preoperative exercise was an effective intervention for reducing cardiac and renal complication rates after surgical interventions and also the length of critical care stay. ${ }^{28}$ Indeed, a forward walking program started before and continued after surgery reduced the hospital stay. ${ }^{30}$

A retrospective cohort with a longer follow-up showed that exercise is an effective intervention to reduce aneurysmal expansion and aortic aneurysm repair rates. ${ }^{32}$ This result is similar to that in an animal model study. ${ }^{41}$ Because these clinical trials had short-term follow-up and low numbers of patients, the effect direction may yet change with the addition of new studies.

Patients with aortic aneurysms have a life expectancy lower than that of individuals of the same age in the same population, ${ }^{17}$ and it has been recognized the exercise decreases mortality in patients with stable coronary heart disease..$^{42}$ Although exercise did not reduce the mortality rates in this review, some mortality can be attributed to patients' associated clinical risk factors. ${ }^{17}$ Exercise could also be advocated to improve patients' quality of life, but data are insufficient to assess this outcome. ${ }^{18}$

With respect to safety concerns, in all studies exercise did not increase the risks of rupture, death, or severe cardiovascular adverse effects. Additionally, there are presumably large numbers of patients with undiagnosed small abdominal aortic aneurysms in exercise programs and rupture rates are low. Indeed, cardiorespiratory fitness is a marker of mortality, ${ }^{43}$ and improved fitness can be a valuable intervention to prevent at least serious complications whenever surgery is necessary. Good fitness levels are considered important to reduce hospital stay with no reduction in surgical mortality rates..$^{33}$ These facts still do not constitute evidence to support recommending exercise to patients with small aneurysms at surveillance, since few patients have been evaluated.

A recent review included five studies and conducted a descriptive analysis. ${ }^{44}$ We decided that two of those studies could not be appropriately included in the systematic review without increasing clinical heterogeneity. One study had no control group, ${ }^{27}$ and the other evaluated respiratory physiotherapy, not exercise. ${ }^{31}$ Another systematic review has problems related to selection since it included the same study three times in the meta-analysis and described surrogate outcomes. ${ }^{45}$

The overall quality of the evidence of this review was graded "very low" because of the use of a rigorous methodology to reduce the risk of bias for clinical trials. A comprehensive and sensitive literature search was carried out, and at least two authors collected, extracted, and assessed the quality of data from studies. Additionally, a validated study was used to determine the risk of bias of the studies included. ${ }^{46}$ Finally, the GRADE approach was used to grade the final quality of the body of the evidence. ${ }^{24}$

There was heterogeneity in the amount, duration, and type of exercise among the studies included, possibly leading to different fitness levels. This heterogeneity could also lead to variation in individuals' physiologic responses. Furthermore, the rate of loss to follow-up was high during the interventions in the clinical trials; however, this was sometimes impossible to avoid (e.g., some patients were withdrawn due to 
acute myocardial infarction and respiratory failure). A per-protocol analysis was thus chosen for analytic purposes. These concerns led us to conclude that the optimal duration and intensity of exercise remain undetermined. Indeed, aortic aneurysms larger than $70 \mathrm{~mm}$ have a lower prevalence but the worst prognosis. ${ }^{47}$ Thus, the evidence is not valid for this subgroup of patients.

One limitation of this review is that most of the studies included were performed in a well-controlled environment, which does not represent everyday life. Indeed, one patient in the intervention group in the study by Kothmann et al..$^{29}$ had ventricular fibrillation and was successfully resuscitated. Aneurysms are more prevalent in men, and no study included a sufficient number of women to arrive at any conclusions for this subgroup, despite the fact that it has worse prognosis. ${ }^{48}$

Whether the effect of intervention is limited to the duration of exercise or can be extended even when a patient becomes sedentary later in life remains unknown. In two clinical trials, no patients were referred for surgery during surveillance, because of the short follow-up period. ${ }^{18,29}$ Additionally, the causes of aneurysm growth are unclear, ${ }^{49}$ and some rapidly expanding aneurysms reach the threshold for surgery before the expected time.$^{50}$ This may indicate the presence of a subgroup of patients with increased exercise-related risks. Thus, to ensure safety, it is essential to set intervals for conducting ultrasound surveillance during exercise periods for patients with both small and large aneurysms.

Aneurysm diameter was imbalanced between intervention and control groups. Because aneurysm growth rate is directly dependent on original aneurysm diameter, ${ }^{11}$ related outcomes (e.g., aneurysm growth rate and rupture) could also be influenced.

Two-thirds of patients in the study by Myers et al. ${ }^{11}$ were not able to achieve the amount and intensity of exercise required for inclusion. Other types, durations, and intensities of exercise might be of value for these patients. Additionally, all studies only evaluated patients with abdominal aortic aneurysms.

There is a glaring need to perform more pragmatic clinical trials with longer follow-ups to achieve a sufficient number of patients to reduce uncertainty. Prospective studies with women are also necessary.

\section{CONCLUSION}

The results of this systematic review and meta-analysis showed that there is very low quality evidence that exercise was effective and safe for patients with asymptomatic aortic aneurysms. Exercise did not impact aneurysm expansion rates. Six weeks of preoperative exercise decreased renal and cardiovascular surgical complications and reduced intensive care unit stays. Preoperative and postoperative forward walking reduced hospital stays. These outcomes need more studies to confirm the potential use of exercise for aortic aneurysm patients, since the quality of the evidence was judged as very low quality for all the outcomes studied.

\section{PERSPECTIVE}

Patients with aortic aneurysms are faced with a dilemma: although exercising could increase the risk of aneurysm rupture, a sedentary lifestyle increases the risk of death, mainly due to coronary artery disease. The prevalence of aortic aneurysms is high in older patients, ${ }^{3}$ but most patients have small abdominal aortic aneurysms with higher mortality rates compared with patients of the same age, depending on the clinical condition. ${ }^{12}$ Therefore, this issue is relevant for patients, exercise professionals, and stakeholders involved in creation of new treatment interventions. To our knowledge, no other systematic review has addressed this issue with the same level of quality. This review revealed no deaths or aneurysm ruptures related to exercise. Additionally, although the clinical trials showed no reduction in aneurysm growth rates, a retrospective cohort with longer follow-up showed reductions in aneurysm growth rate and in the number of patients referred for surgery. This evidence demonstrates reductions in cardiac and renal complication rates, hospital stays, and intensive care unit stays. The present review identifies a new patient population in whom the benefits of exercise should be studied. While the general population experiences increased quality and quantity of life from exercising, patients with aneurysms might benefit from exercise as a treatment option.

\section{ACKNOWLEDGMENTS}

We thank Paulo Minalli, physical educator and content specialist, for helping us to understand contexts, definitions, and outcomes. We also thank Angela Morben, DVM, ELS, from Edanz Group (www.edanzediting.com/ac), for editing a draft of this manuscript.

\section{REFERENCES}

1. Johnston KW, Rutherford RB, Tilson MD, Shah DM, Hollier L, StanleyJC. Suggested standards for reporting on arterial aneurysms. Subcommittee on Reporting Standards for Arterial Aneurysms, Ad Hoc Committee on Reporting Standards, Society for Vascular Surgery and North American Chapter, International Society for Cardiovascular Surgery. J Vasc Surg. 1991;13(3):452-8. http:// dx.doi.org/10.1067/mva.1991.26737. PMid:1999868. 
2. Moll FL, Powell JT, Fraedrich G, et al. Management of abdominal aortic aneurysms clinical practice guidelines of the European Society for Vascular Surgery. Eur J Vasc Endovasc Surg. 2011;41(Suppl 1):S158. http://dx.doi.org/10.1016/j.ejvs.2010.09.011. PMid:21215940.

3. Singh K, Bønaa KH, Jacobsen BK, Bjørk L, Solberg S. Prevalence of and risk factors for abdominal aortic aneurysms in a populationbased study: the Tromsø Study. Am J Epidemiol. 2001;154(3):236-44 http://dx.doi.org/10.1093/aje/154.3.236. PMid:11479188.

4. Scott RA, Wilson NM, Ashton HA, Kay DN. Influence of screening on the incidence of ruptured abdominal aortic aneurysm: 5-year results of a randomized controlled study. Br J Surg. 1995;82(8):106670. http://dx.doi.org/10.1002/bjs.1800820821. PMid:7648155.

5. Aggarwal S, Qamar A, Sharma V, Sharma A. Abdominal aortic aneurysm: a comprehensive review. Exp Clin Cardiol. 2011;16(1):115. PMid:21523201.

6. Cosford PA, Leng GC. Screening for abdominal aortic aneurysm Cochrane Database Syst Rev. 2007;(2):CD002945. PMid:17443519.

7. Lederle FA. The natural history of abdominal aortic aneurysm. Acta Chir Belg. 2009;109(1):7-12. http://dx.doi.org/10.1080/000 15458.2009.11680364. PMid:19341189.

8. Mell M, White JJ, Hill BB, Hastie T, Dalman RL, Stanford Abdominal Aortic Aneurysm Specialized Center of Clinically Oriented Research Investigators. No increased mortality with early aortic aneurysm disease. J Vasc Surg. 2012;56(5):1246-5. http://dx.doi.org/10.1016/j. jvs.2012.04.023. PMid:22832264.

9. Reed WW, Hallett JW Jr, Damiano MA, Ballard DJ. Learning from the last ultrasound. A population-based study of patients with abdominal aortic aneurysm. Arch Intern Med. 1997;157(18):20648. http://dx.doi.org/10.1001/archinte.1997.00440390050007. PMid:9382661.

10. The UK Small Aneurysm Trial Participants. Mortality results for randomised controlled trial of early elective surgery or ultrasonographic surveillance for small abdominal aortic aneurysms. Lancet. 1998;352(9141):1649-55. http://dx.doi.org/10.1016/S01406736(98)10137-X. PMid:9853436.

11. Myers J, Mcelrath $M$, Jaffe $A$, et al. A randomized trial of exercise training in abdominal aortic aneurysm disease. Med Sci Sports Exerc. 2014;46(1):2-9. http://dx.doi.org/10.1249/MSS.0b013e3182a088b8. PMid:23793234.

12. Newman AB, Arnold AM, Burke GL, O'Leary DH, Manolio TA. Cardiovascular disease and mortality in older adults with small abdominal aortic aneurysms detected by ultrasonography: the cardiovascular health study. Ann Intern Med. 2001;134(3):182-90. http://dx.doi.org/10.7326/0003-4819-134-3-200102060-00008. PMid:11177330.

13. Brady AR, Fowkes FG, Greenhalgh RM, Powell JT, Ruckley CV, Thompson SG. Risk factors for postoperative death following elective surgical repair of abdominal aortic aneurysm: results from the UK Small Aneurysm Trial. Br J Surg. 2000;87(6):742-9. http:// dx.doi.org/10.1046/j.1365-2168.2000.01410.x. PMid:10848851.

14. Garber CE, Blissmer B, Deschenes MR, et al. American College of Sports Medicine position stand. Quantity and quality of exercise for developing and maintaining cardiorespiratory, musculoskeletal, and neuromotor fitness in apparently healthy adults: guidance for prescribing exercise. Med Sci Sports Exerc. 2011;43(7):1334-59. http://dx.doi.org/10.1249/MSS.0b013e318213fefb. PMid:21694556.

15. Almeida OP, Khan KM, Hankey GJ, Yeap BB, Golledge J, Flicker L. 150 minutes of vigorous physical activity per week predicts survival and successful ageing: a population-based 11-year longitudinal study of 12201 older Australian men. Br J Sports Med. 2014;48(3):220-5. http://dx.doi.org/10.1136/bjsports-2013-092814. PMid:24002240.
16. Caspersen C), Powell KE, Christenson GM. Physical activity, exercise, and physical fitness: definitions and distinctions for health-related research. Public Health Rep. 1985;100(2):126-31. PMid:3920711.

17. Chaikof EL, Dalman RL, Eskandari MK, et al. The Society for Vascular Surgery practice guidelines on the care of patients with an abdominal aortic aneurysm. J Vasc Surg. 2018;67(1):2-77.e2. http://dx.doi.org/10.1016/j.jvs.2017.10.044. PMid:29268916.

18. Tew GA, Moss J, Crank H, Mitchell PA, Nawaz S. Endurance exercise training in patients with small abdominal aortic aneurysm: a randomized controlled pilot study. Arch Phys Med Rehabil. 2012;93(12):2148-53. http://dx.doi.org/10.1016/j.apmr.2012.07.012. PMid:22846453.

19. Higgins JPT, Green S. Cochrane handbook for systematic reviews of interventions. Version 5.1.0. USA: The Cochrane Collaboration; 2011.

20. Moher D, Liberati A, TetzlaffJ, Altman DG, PRISMA Group. Preferred reporting items for systematic reviews and meta-analyses: the PRISMA statement. Int J Surg. 2010;8(5):336-41. http://dx.doi. org/10.1016/j.jijsu.2010.02.007. PMid:20171303.

21. Oliveira R, Nakajima E, Riera R, Baptista-Silva J. Physical exercises for aortic aneurysm: a systematic review. United Kingdom: NIHR; 2016. PROSPERO 2016 CRD42016041468. [cited 2019 aug 15]. https://www.crd.york.ac.uk/prospero/display_record. php?ID=CRD42016041468

22. Ouzzani M, Hammady H, Fedorowicz Z, Elmagarmid A. Rayyan-a web and mobile app for systematic reviews. Syst Rev. 2016;5(1):210. http://dx.doi.org/10.1186/s13643-016-0384-4. PMid:27919275.

23. Manager R. (RevMan) Version 5.3 [software]. Copenhagen: The Nordic Cochrane Centre, The Cochrane Collaboration; 2014.

24. GRADEpro GDT. GRADEpro Guideline Development Tool [software]. Hamilton: McMaster University; 2015.

25. Lima RM, Vainshelboim B, Ganatra R, Dalman R, Chan K, Myers J. Exercise training improves ventilatory efficiency in patients with a small abdominal aortic aneurysm: A randomized controlled study. J Cardiopulm Rehabil Prev. 2018;38(4):239-45. http://dx.doi. org/10.1097/HCR.0000000000000270. PMid:28727673.

26. Myers JN, White JJ, Narasimhan B, Dalman RL. Effects of exercise training in patients with abdominal aortic aneurysm: preliminary results from a randomized trial. J Cardiopulm Rehabil Prev. 2010;30(6):374-83. http://dx.doi.org/10.1097/HCR.0b013e3181ebf2db. PMid:20724934.

27. Barakat HM, Shahin Y, Barnes R, et al. Supervised exercise program improves aerobic fitness in patients awaiting abdominal aortic aneurysm repair. Ann Vasc Surg. 2014;28(1):74-9. http://dx.doi. org/10.1016/j.avsg.2013.09.001. PMid:24332259.

28. Barakat HM, Shahin Y, Khan JA, McCollum PT, Chetter IC. Preoperative supervised exercise improves outcomes after elective abdominal aortic aneurysm repair. Ann Surg. 2016;264(1):47-53. http://dx.doi. org/10.1097/SLA.0000000000001609. PMid:26756766.

29. Kothmann E, Danjoux G, Owen SJ, Parry A, Turley AJ, Batterham AM. Reliability of the anaerobic threshold in cardiopulmonary exercise testing of patients with abdominal aortic aneurysms. Anaesthesia. 2009;64(1):9-13. http://dx.doi.org/10.1111/j.13652044.2008.05690.x. PMid:19086999.

30. Wnuk BR, Durmała J, Ziaja K, Kotyla P, Woźniewski M, Błaszczak E. A controlled trial of the efficacy of a training walking program in patients recovering from abdominal aortic aneurysm surgery. Adv Clin Exp Med. 2016;25(6):1241-371. http://dx.doi.org/10.17219/ acem/62239. PMid:28028979.

31. Dronkers J, Veldman A, Hoberg E, van der Waal C, van Meeteren N. Prevention of pulmonary complications after upper abdominal surgery by preoperative intensive inspiratory muscle training: a 
randomized controlled pilot study. Clin Rehabil. 2008;22(2):134-42. http://dx.doi.org/10.1177/0269215507081574. PMid:18057088.

32. Nakayama A, Morita $H$, Nagayama $M$, et al. Cardiac rehabilitation protects against the expansion of abdominal aortic aneurysm. J Am Heart Assoc. 2018;7(5):e007959. http://dx.doi.org/10.1161/ JAHA.117.007959. PMid:29487112.

33. Hayashi K, Hirashiki A, Kodama A, et al. Impact of preoperative regular physical activity on postoperative course after open abdominal aortic aneurysm surgery. Heart Vessels. 2016;31(4):57883. http://dx.doi.org/10.1007/s00380-015-0644-6. PMid:25666952.

34. Bailey TG, Perissiou M, Windsor MT, et al. Effects of acute exercise on endothelial function in patients with abdominal aortic aneurysm. Am J Physiol Heart Circ Physiol. 2018;314(1):H19-30. http://dx.doi. org/10.1152/ajpheart.00344.2017. PMid:28939648.

35. Weston M, Batterham AM, Tew GA, et al. Patients awaiting surgical repair for large abdominal aortic aneurysms can exercise at moderate to hard intensities with a low risk of adverse events. Front Physiol. 2016;7:684. PMid:28119627.

36. ClinicalTrials.gov. Surviving aneurysm surgery: a pilot study on exercise training in abdominal aortic aneurysm patients. USA: $U$. S. National Library of Medicine; 2017. NCT01805973.

37. ClinicalTrials.gov. The assessment of the feasibility of a home based exercise programme in the older patient following major surgery. USA: U. S. National Library of Medicine; 2017. NCT03064308.

38. ClinicalTrials.gov. Prehabilitation for aortic repair patients - full text view. USA: U. S. National Library of Medicine; 2017. NCT02767518.

39. Mosk A. Pre operative optimisation pathway for elderly surgical patients - OUTO. The Netherlands: Netherlands Trial Register. NTR5932.

40. Senn S. Testing for baseline balance in clinical trials. Stat Med. 1994;13(17):1715-26. http://dx.doi.org/10.1002/sim.4780131703. PMid:7997705.

41. Nakahashi TK, Hoshina K, Tsao PS, et al. Flow loading induces macrophage antioxidative gene expression in experimental aneurysms. Arterioscler Thromb Vasc Biol. 2002;22(12):201722. http://dx.doi.org/10.1161/01.ATV.0000042082.38014.EA PMid:12482828.

42. Stewart RAH, Held C, Hadziosmanovic N, et al. Physical activity and mortality in patients with stable coronary heart disease. J Am Coll Cardiol. 2017;70(14):1689-700. http://dx.doi.org/10.1016/j. jacc.2017.08.017. PMid:28958324.

43. Kodama S, Saito K, Tanaka S, et al. Cardiorespiratory fitness as a quantitative predictor of all-cause mortality and cardiovascular events in healthy men and women. JAMA. 2009;301(19):2024-35 http://dx.doi.org/10.1001/jama.2009.681. PMid:19454641.

44. Pouwels S, Willigendael EM, van Sambeek MR, Nienhuijs SW, Cuypers PW, Teijink JA. Beneficial effects of pre-operative exercise therapy in patients with an abdominal aortic aneurysm: a systematic review. Eur J Vasc Endovasc Surg. 2015;49(1):66-76. http://dx.doi. org/10.1016/j.ejvs.2014.10.008. PMid:25457300.

45. Kato M, Kubo A, Green FN, Takagi H. Meta-analysis of randomized controlled trials on safety and efficacy of exercise training in patients with abdominal aortic aneurysm. J Vasc Surg. 2019;69(3):933-43. http://dx.doi.org/10.1016/j.jvs.2018.07.069. PMid:30578072.
46. Higgins JP, Altman DG. Assessing risk of bias in included studies. In: Higgins JP, Green S, editors. Cochrane Handbook for Systematic Reviews of Interventions Version 5.1.0. USA: The Cochrane Collaboration; 2011.

47. Conway KP, Byrne J, Townsend M, Lane IF. Prognosis of patients turned down for conventional abdominal aortic aneurysm repair in the endovascular and sonographic era: Szilagyi revisited? J Vasc Surg. 2001;33(4):752-7. http://dx.doi.org/10.1067/mva.2001.112800. PMid:11296328.

48. Norman PE, Powell JT. Abdominal aortic aneurysm: the prognosis in women is worse than in men. Circulation. 2007;115(22):28659. http://dx.doi.org/10.1161/CIRCULATIONAHA.106.671859. PMid:17548742.

49. Brewster DC, Cronenwett JL, Hallett JW Jr, et al. Guidelines for the treatment of abdominal aortic aneurysms: report of a subcommittee of the Joint Council of the American Association for Vascular Surgery and Society for Vascular Surgery. J Vasc Surg. 2003;37(5):1106-17. http://dx.doi.org/10.1067/mva.2003.363. PMid:12756363.

50. Scott RA, Tisi PV, Ashton HA, Allen DR. Abdominal aortic aneurysm rupture rates: a 7-year follow-up of the entire abdominal aortic aneurysm population detected by screening. J Vasc Surg. 1998;28(1):124-8. http://dx.doi.org/10.1016/S0741-5214(98)702071. PMid: 9685138.

Correspondence Vladimir Tonello de Vasconcelos Universidade Federal de São Paulo - UNIFESP, Departamento de Medicina

Rua Borges Lagoa, 754 CEP 04038-001 - São Paulo (SP), Brasil Tel.: +55 (11) 5576-4848 E-mail:vtvascon@hotmail.com

Author information RAO - Professor, Vascular Surgery, Universidade Federal de Uberlândia; post-graduation student, Universidade Federal de São Paulo (UNIFESP)

EN - Cardiologist, post-graduation student, Universidade Federal de São Paulo (UNIFESP). VTV and JCCBS - Professor, Vascular Surgery, Universidade Federal de São Paulo (UNIFESP). RR - Professor, Evidence Based Medicine, Universidade Federal de São Paulo (UNIFESP).

Author contributions Conception and design: RAO, RR, JCCBS Analysis and interpretation: RAO, EN, VTV, RR, JCCBS Data collection: RAO, EN, VTV Writing the article: RAO, VTV, RR, JCCBS Critical revision of the article: RAO, VTV, RR, JCCBS Final approval of the article*: RAO, EN, VTV, RR, JCCBS Statistical analysis: RAO, VTV, RR, JCCBS Overall responsibility: RAO, EN, VTV, RR, JCCBS

*All authors have read and approved of the final version of the article submitted to J Vasc Bras. 